\title{
AuCPace: Efficient verifier-based PAKE protocol tailored for the IloT
}

\author{
Björn Haase and Benoît Labrique \\ Endress+Hauser Conducta GmbH\&Co. KG, Germany \\ bjoern.haase@endress.com
}

\begin{abstract}
Increasingly connectivity becomes integrated in products and devices that previously operated in a stand-alone setting. This observation holds for many consumer applications in the so-called "Internet of Things" (IoT) as well as for corresponding industry applications (IIoT), such as industrial process sensors. Often the only practicable means for authentication of human users is a password. The security of password-based authentication schemes frequently forms the weakest point of the security infrastructure.

In this paper we first explain why a tailored protocol designed for the IIoT use case is considered necessary. The differences between IIoT and the conventional Internet use-cases result in largely modified threats and require special procedures for allowing both, convenient and secure use in the highly constrained industrial setting.

Specifically the use of a verifier-based password-authenticated key-exchange (V-PAKE) protocol as a hedge against public-key-infrastructure (PKI) failures is considered important. Availability concerns for the case of failures of (part of) the communication infrastructure makes local storage of access credentials mandatory. The larger threat of physical attacks makes it important to use memory-hard password hashing.

This paper presents a corresponding tailored protocol, AuCPace, together with a security proof within the Universal Composability (UC) framework considering fully adaptive adversaries. We also introduce a new security notion of partially augmented PAKE that provides specific performance advantages and makes them suitable for a larger set of IIoT applications.

We also present an actual instantiation of our protocol, AuCPace25519, and present performance results on ARM Cortex-M0 and Cortex-M4 microcontrollers. Our implementation realizes new speed-records for PAKE and X25519 Diffie-Hellman for the ARM Cortex M4 architecture.
\end{abstract}

Keywords: Password Authenticated Key Exchange, V-PAKE, PAKE, elliptic curves, Cryptographic Protocols, Universal Composability, IEC-62443, Industrial Control, Curve25519, X25519

\section{Introduction}

Since recently, wireless and networking technology becomes integrated in products and devices that previously operated in a stand-alone setting, both in consumer applications in the so-called "Internet of Things" (IoT) as well as in the corresponding industry setting, the "Industrial IoT" (IIoT). Often communication technology and security protocols are employed that were not originally tailored and designed for the resource-constrained setting and the specific threat model.

In comparison to conventional un-connected devices, security becomes a crucial aspect to consider, specifically in the IIoT. Often, users tend to use short and easily memorable passwords. For this reason emerging industry standards, such as from the IEC-62443 
family, rightfully require two-factor authentication for higher security levels (SL-3, SL-4). Still then, however, suitable protection of the second factor "password" remains important.

Today, in most Internet communication and many IIoT applications protocols such as TLS based web servers are used, that were not originally designed for the IIoT use-case. Mostly, a trustworthy and failure-free Public-Key-Infrastructure (PKI) is indispensable for providing even basic protection of passwords, e.g., against phishing or man-in-the-middle attacks. However in the IIoT setting, specifically for industrial installations not fully operating according to standards such as IEC-62443, today integration of the devices in a PKI is not always available.

\subsection{Note on the current standardization activities for industrial instal- lations}

Security is a new topic for most industry installations and component suppliers. For the project of securing industrial installations, suppliers and implementers currently seem to first concentrate on standardizing the security protocols of the machine-machine interfaces. User authentication tends to be addressed in a second step only.

One example is the Common Industrial Protocol (CIP Security) standard family which forms the base for the EtherNet/IP(tm) and DeviceNet(tm) standards. Here, while the standardization of security mechanisms for the machine-machine interfaces is widely settled, the standardization of user authentication as part of the "CIP Authorization Profile" is still a work in progress.

In our assessment, we consider user authentication to be as critical as machine-machine authentication. In many settings the attacker is given equivalent power over an installation by gaining control over human machine interfaces. With this paper we also aim at contributing to the project of resolving this issue.

\subsection{Differences between conventional web security and IloT}

One of the most remarkable differences between the conventional web server setting (here referred to as "web shop" use case) and the typical IIoT setting corresponding, e.g., to an industrial plant is illustrated in Figure 1. In the former case few servers, e.g., web servers, interface to many clients, e.g., web browsers which come with pre-installed configuration for certificate authorities trusted by the browser supplier. In the latter case, one single client, e.g., a tablet-computer based human machine interface (HMI), might be used for configuring many servers, e.g., sensors or control valves.

Unlike in the "web shop" use case, in the IIoT setting a dramatically larger number of server certificates needs to be configured and maintained. Often self-signed certificates are used for servers, leading to the significant risk that the essential corresponding configurations on the client side (e.g., browsers) are omitted. Operators and system administrators used to the more convenient "web shop" setting might not even be aware that such configuration is mandatory for security.

As a consequence, the threat of PKI failures should be considered very carefully for remote HMI access to (I)IoT units. This is one of the reasons, why since recently strategies for password protection as a hedge against PKI failures have regained academic and industrial interest [JKX18, PW17, HL17].

Moreover many IIoT devices, notably battery-driven devices, will not be permanently "online". Availability concerns for the case of (partial) failure of networking infrastructure often make it mandatory to locally store user credentials, typically in unprotected memory. Furthermore, IoT devices might be much more exposed to physical attacks. For all of these reasons, protection of passwords forms a crucial point of any (I)IoT security solution. 


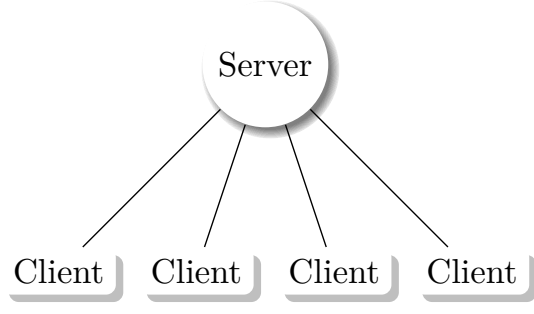

Web-Shop use-case

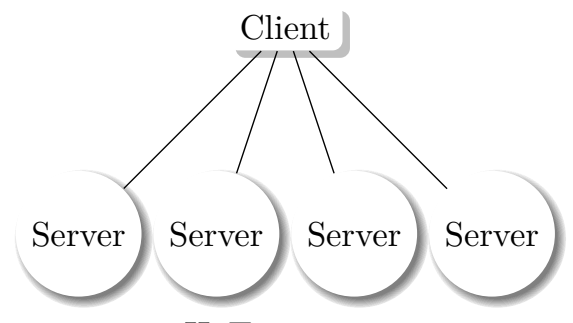

IIoT use-case

Figure 1: Use-cases for conventional Internet applications and IIoT.

\subsection{Strategies for protecting passwords}

For the protection of passwords two complementary approaches could be distinguished. On the one hand, memory-hard password hash algorithms such as scrypt [PJ12] and Argon2 [BDKJ16] aim at increasing the cost of offline dictionary attacks. On the other hand password-based key exchange (PAKE) protocols allow for establishing a secure, high-entropy shared session key over an insecure communication channel. This holds even if only a low-entropy secret key, the password $p w$ is shared (see, e.g., [Gre18] for a recent gentle introduction to the concept of PAKE).

One of the important advantages of PAKE protocols published since the early works by Bellovin and Merritt and Jablon [BM92, Jab96] is the fact that neither a public-key infrastructure (PKI) nor a trusted hardware component capable of securely storing highentropy keys is required as prerequisite. PAKE protocols, thus, match very closely the needs of the IIoT use-case.

PAKE protocols essentially come in two variants. Firstly, so-called balanced or symmetric PAKE protocols, for instance [BM92, $\mathrm{CHK}^{+}$05, BFK09, Jab96, BMP00], are designed such that both parties, initiator and responder, require that the same password $p w$ is available on both sides. Secondly, so-called verifier-based PAKE protocols (also known as V-PAKE, asymmetric or augmented PAKE) protocols could be distinguished, where the server is given access only to a password-verifier $W$ and the clear-text password is only available to the client party, e.g., [Wu98, PW17, GMR06, Jab97]. V-PAKE allows for some limited additional protection for the case that the attacker gets access to the server's password-verifier database. The additional protection due to augmentation should not be over-estimated but still could make the task significantly more difficult for the attacker. In all known protocols V-PAKE comes with significant additional computational overhead in comparison to PAKE.

A peculiarity in the industrial IIoT setting is that we should be expecting to find the very same password in use on many small devices. Due to password re-use the compromise of one small server might affect the security of a larger infrastructure. On the other hand, availability concerns for the case of failures of (part of) the network infrastructure often make it mandatory to locally store this sensitive data and often no protected storage media, such as smart-card circuits, are available. For this reason verifier-based PAKE using memory hard password hashing usually should be considered to provide the best practically feasible security strategy regarding HMI authentication for IIoT applications.

\subsection{Why industrial instrumentation needs a specially tailored V-PAKE protocol}

Unfortunately, it is not uncommon that IoT and industrial devices have only very limited energy and computational resources available, specifically if they have to be conformable with the constraint set that applies for intrinsically safe explosion protection (IEC60079-11) 


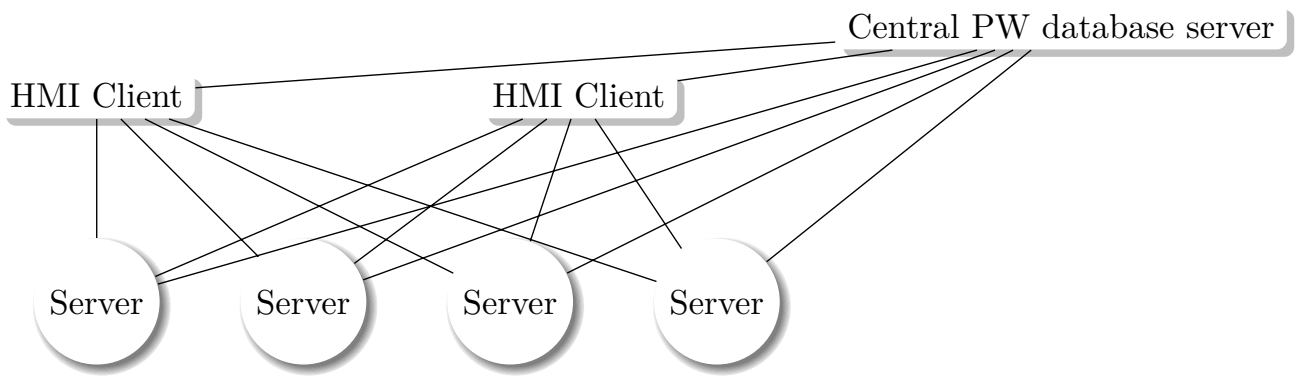

Figure 2: IIoT setting with central password distribution infrastructure.

\section{[HL17].}

For important classes of devices, use of memory-hard password hashing is precluded due to the limited memory and computational capacities. Also the computational complexity of some established V-PAKE protocols that were originally developed for office information technology might prevent actual use in the IIoT. Note that all of the most efficient known augmented protocols (requiring three exponentiations) or components of the protocols, such as AugPake [SKI10] or OPAQUE [JKX18] are covered by patents. It is important to consider that also devices even smaller than typical IIoT devices need sound password protection, such as, e.g., legacy fieldbus systems with bluetooth-based wireless HMI interfaces [HL17].

In all of these settings, efficiency considerations and pending patents will be a crucial factor ultimately deciding upon whether or not sound password protection could actually be implemented by manufacturers. Note that specifically for the smallest devices such as, e.g., temperature sensors, a significant commercial cost constraint applies. Power efficiency, code size and ease-of-implementation are crucial factors that will be decisive for actual roll-out of a more secure or insecure solution, i.e., efficiency is of utmost importance.

Regarding efficiency of V-PAKE solutions, optimizations need to apply on all levels of the security implementation, protocol design, algorithmic optimizations (e.g., group operations on elliptic curves) and low-level arithmetic. Unfortunately, most protocols today were mainly designed with typical office environments in mind.

It is also important to consider that authentication protocols are often embedded in a larger system, involving, e.g., protocols allowing for distributing password verifiers to server entities from a central management infrastructure as sketched in figure 2. Note that in the IEC-62443-4-2 draft standard, centralized user account management systems are declared mandatory even at the lowest security level SL-1. This provides significant challenges for all devices that are not permanently "on-line" or isolated by purpose from the main company network, i.e., in settings where a direct network connection to a central password database server may be not reliably available.

\subsection{Contribution of this paper}

This paper aims at contributing to the project of securing IIoT applications by comprehensively addressing all of efficiency, implementation and patent issues. We aim at doing so by introducing an efficiency-optimized V-PAKE protocol "Augmented Composable Password Authenticated Connection Establishment" (AuCPace) and an actual instantiation AuCPace25519 that is, to the best of our knowledge, freely usable without inflicting intellectual property rights and specifically tailored for the exact subset of devices where security is most likely to be discarded: Small extremely resource-constrained and/or low-cost devices where integrating sound security is particularly difficult.

The project of protecting industry installations clearly requires considering a larger scope, such as sketched in figure 2. For the scope of this work, however, we identified the 
need to concentrate in a first step on the sub-component of the authentication protocol running between the HMI server and client units, i.e., leaving out the significant complexity of the user credential distribution process for local password registration.

Our scheme arranges for use of memory-hard password hashes also on smallest devices with little memory, since it is deferring the costly password hashing to the client entities.

Our work builds up upon the work in [HL17] by explicitly providing formal security proofs for the specific optimized design choices therein, e.g., regarding the cheaper alternative to conventional point verification employing twist security [BL19] and avoidance of costly and memory-consuming point compression. Note that such aspects are usually not relevant for the conventional office IT setting.

We do so by providing a security proof for AuCPace in the UC framework with joint state [CR03]. We base our analysis on the UC-based security model of [GMR06]. We show that our protocol, unlike most Diffie-Hellman-based constructions, could be proven secure in the UC model, even when considering fully adaptive adversaries. We extend this model for allowing a new operation mode that we coined partially augmented PAKE. We show how it is possible to implement AuCPace in its partially augmented variant without any computational overhead in comparison to conventional "balanced" PAKE for the resource-constrained server, while maintaining all of the most relevant security guarantees in the IIoT setting.

Our protocol could be clearly modularized into 1.) a balanced sub-protocol coined CPace (Composable Pace) which shares important design features with PACE [BFK09] and 2.) an augmentation layer allowing for both conventional augmentation and our newly introduced partial augmentation.

As one concrete instantiation we present a protocol AuCPace25519 which adds further optimizations also on the group arithmetic and field arithmetic level. We present performance results for both, AuCPace and Diffie-Hellman protocols for ARM Cortex M0 and Cortex M4 microcontrollers.

We hope that the new speed-records for constant-time implementation of both, PAKE and the X25519 Diffie-Hellman Protocol on the ARM Cortex M4, that we report in this paper will make it possible to enlarge the set of targets that could afford integrating state-of-the-art security technology.

\subsection{Organization of this paper}

For this paper we consider different groups of readers being more or less inclined to theoretical analysis and actual implementation on constrained targets respectively. For this reason, this paper is organized as follows.

In section 2 we first review related work on PAKE protocols and the definitions of security used for their respective analysis. Since AuCPace may be considered a SPEKE [Jab96] variant, we will explain, why we chose SPEKE as basis despite the fact that for SPEKE no proof is available, when used with elliptic curves. We then give a short introduction to the concepts and methods used for proofs in the framework of Universal Composability (UC). In section 2 we also will give a short introduction to the concept of "partial augmentation" which will be introduced more formally only later as part of the security proofs. We do so in order to make the paper self-contained also for readers willing to skip the security proofs in a first step.

Subsequently, in section 3 we will introduce the full protocol AuCPace. There we will also explain the design guidelines that motivated specific choices and constructions.

We then present the proofs, first handling the case of the balanced component CPace (section 4). In section 5 we then use the composition theorem for proving security of our V-PAKE construction. In the subsequent section 6 we introduce the concept of "partial augmentation" and show how to essentially halve the server's computational complexity. In section 7 we compare our proposal AuCPace with other efficient PAKE constructions. 
Subsequently in section 8 we describe the implementation strategy on Cortex M0 and M4 microcontrollers for our reference implementation AuCPace25519. We conclude the paper by presenting actual performance benchmarks on different microcontrollers and embedded bluetooth-transceiver platforms in section 9 .

Major parts of the optimized low-level code presented in this paper will be made freely available under a $\mathrm{CC} 0$ license at [HL18].

In the appendix we present an outline, how a variant of our protocol could also be implemented on conventional elliptic curves in short Weierstrass form and how the very similar balanced protocol from [HL17] could be proven secure in the UC model.

\section{Review of PAKE protocols and their security analysis}

\subsection{Overview on PAKE protocols}

Despite the clear-cut security advantages, there are a couple of reasons that hampered use of PAKE protocols in a number of applications [EKSS09]. Here patent pitfalls did play a major role. Notably, EKE [BM92] and SPEKE [Jab96] were patented until very recently and also some of the most efficient protocols in the IEEE 1363 standard family are patented. This resulted in protocols such as SRP[Wu98], J-PAKE [HR10] and PACE [BFK09] which did include additional complexity solely for patent circumvention.

As a result of patent circumvention, a large number of different PAKE protocols have been presented. This did hamper thorough security analysis. Firstly proofs became more complex or impractical because of the additional complexity for patent circumvention. Secondly the number of protocol variants grew, reducing the amount of analysis effort spent on each candidate.

Since the SPEKE patent has expired recently, some of the circumventions used for PACE [BFK09] became obsolete. CPace, as presented in this paper, has been developed in the process of removing patent circumvention steps. We did observe that the resulting changes allowed for a natural way of agreeing on a session id before entering the protocol and thus opened a path for a proof strategy within the UC framework.

Many protocols, including EKE and SPEKE, have been first suggested without a formal security proof. Doing so comes at the risk of accidentally including serious design-flaws. Many PAKE protocols have later been shown to be insecure. A recent example showing the need of thorough security analysis is the case of zkPAKE presented in [MRA15] that has been shown vulnerable to offline guessing attacks in [BRSS18].

For a recent comprehensive overview over different PAKE protocols and proof strategies see, e.g., [SOAA15].

\subsection{Security guarantees of PAKE and V-PAKE protocols}

All types of PAKE and V-PAKE protocols base security on a low-entropy secret, the password. The direct consequence is that they could not unconditionally be proven secure because in any case exhaustive or dictionary searches by so-called "online attacks" could not be prevented. For each "online" session with a server an attacker always could test at least one password. Due to the low entropy this attack should always be assumed to succeed.

Online attacks could however be mitigated by rate-limiting countermeasures, e.g., by wait times after observing failed login attempts. This countermeasure could not be applied if the authentication protocol allows for "offline" password tests, as, e.g., in case of weak challenge-response protocols.

The security models for PAKE and V-PAKE, thus aim at proving that the "online attack" is the best available attack strategy and that at most one password per online 
session could be tested by the adversary.

V-PAKE goes one step further than balanced PAKE by additionally considering the case that an attacker might get access to the password database of a server by "compromising" it. This type of attack should be considered highly relevant for (I)IOT devices because often the password database is stored in unprotected memory. Unlike in a "server-room setting" an attacker might more easily gain physical access.

In the event of a server compromise it is technically infeasible to prevent offline attacks. Here V-PAKE protocols aim at forcing the adversary to additionally mount an offline search, i.e., the attacker is required to successfully mount both, server compromise and offline dictionary attacks for a successful impersonation.

As a consequence a real security improvement could actually be realized only if the V-PAKE protocol is used in conjunction with dedicated (specifically with memory-hard) password hashes that slow down an offline search.

For most augmented PAKE protocols, it is possible to interleave the substep of offline dictionary attack with the substep of server compromise, e.g., by pre-computing so-called rainbow tables. In [JKX18] Jarecki, Krawczyk and Xu have introduced the notion of "pre-computation attack resistance" of a so-called strong V-PAKE protocol that allows an attacker to mount the offline search only after having successfully compromised the server.

In this paper we additionally introduce the notion of a "partially augmented" PAKE protocol. This notion becomes meaningful in a setting where several servers share the same password database. While for the compromised server, a partially augmented protocol provides guarantees similar to a balanced PAKE protocol, it preserves the security guarantees of a V-PAKE protocol for the remaining subset of servers.

\subsection{Security models}

One important approach for analyzing a PAKE is the game-based "real-or-random" (ROR) model by Bellare, Pointcheval and Rogaway (BPR) [BPR00]. This was later extended to the so-called "find-then-guess" (FTG) model [AFP05]. Simulation-based proof techniques, were established, e.g., by Boyko, MacKenzie and Patel (BMP) in [BPR00].

In 2005 Canetti, Halevi, Katz, Lindell and MacKenzie [CHK $\left.{ }^{+} 05\right]$ have suggested an alternative approach, based on the framework of Universal Composability (UC) [Can01], specifically in its joint-state version [CR03]. It has been shown that UC-secure PAKE constructions could not be realized without either, idealized assumptions, such as random oracles or a common reference string $\left[\mathrm{CHK}^{+} 05\right]$. One of the advantages of analyzing PAKE protocols in the UC framework is that no assumptions regarding the password distribution apply. Related passwords or mistyped related passwords and forward secrecy are inherently also considered. For this reason, the UC-based approach is considered to be providing particularly strong security guarantees [PW17, JKX18].

\subsection{Review of SPEKE and SPEKE variants}

SPEKE [Jab96] was one of the earliest published protocols. The first security analysis of SPEKE has been given by MacKenzie within the BMP simulation based model [Mac01]. Later it has been shown that in fact multiple password guessing with one impersonation is actually feasible [Zha04].

SPEKE variants inherit the property from Diffie-Hellman key exchange, that a manin-the-middle attacker has the possibility to modify both honest parties' resulting DH keys unless the whole transcript of the communication is used for generating the session key [HS14]. If the attacker replaces both intermediate exponentiations $B^{a}$ and $B^{b}$ of the honest participants by a fixed power $\left(B^{a}\right)^{c}$ and $\left(B^{b}\right)^{c}$ both parties will still obtain the same key. Note that this "attack" does not actually affect security in practice [HS14]. 
The original SPEKE protocol suffers from a weakness allowing for simultaneously testing more than one password per online connection [Zha04]. As a mitigation, hashing of the password was suggested in [Jab97], however without fully resolving the problem [LW15].

Unfortunately, the security proofs for SPEKE do not cover the case of elliptic curves. However, SPEKE-related constructions TBPEKE [PW17] and PACE [BFK09] have been proven secure, however those require more exponentiations. It was sometimes conjectured that SPEKE provides forward secrecy, but according to our knowledge this has not been formally proven.

One notable advantage of SPEKE and some SPEKE variants such as PACE [BFK09] when implemented on elliptic curves with integrated mapping [CGIP12] is that no full group operations are required, e.g., allowing also for so-called $x$-coordinate-only implementations. This feature provides some advantages of SPEKE and SPEKE variants in comparison with, e.g., SPAKE [AP05] and its variants and PAK[BMP00]. This way no full group operations are required for implementations but only a less-strict notion of a group modulo negation, that allows for scalar multiplication and differential addition but not for arbitrary point additions.

Verifier-based variants of SPEKE have been suggested already in 1997 [Jab97], unfortunately without a corresponding proof.

\subsection{Review of the UC framework}

In this paper we assume some familiarity with the framework of Universal Composability (UC). As a short introduction, we will give a summary of the essence here. For more details, we refer the reader to [Can00].

The general idea of UC is to define security in terms of idealized functionalities $\mathcal{F}$ which provide services to a set of players $P_{i}$. Moreover the framework considers an adversary $\mathcal{A}$ and an environment $\mathcal{Z}$ and a real-world protocol $\pi$ whose security is to be analyzed. In the context of $\mathrm{UC}$ all of the algorithmic strategy of $\mathcal{A}, \mathcal{Z}$ and $\pi$ are provided in form of code for an interactive Turing machine (ITM). In an actual real-world execution, a plurality of interactive Turing machine instances (ITI) is generated upon request of the environment $\mathcal{Z}$. For instance several ITI may execute the ITM algorithm $\pi$ for the parties $P_{i}$. Also the environment $\mathcal{Z}$ and the adversary $\mathcal{A}$ are given their respective ITI instance.

In the UC framework this "real-world" case is compared with an "ideal-world" case where the protocol $\pi$ is replaced with the ideal functionality $\mathcal{F}$ and the real-world adversary $\mathcal{A}$ with an ideal-world adversary $\mathcal{S}$. The security model is based on the observation that if any (polynomially bounded) environment algorithm $\mathcal{Z}$ cannot distinguish between the "real" and "ideal" world executions with any significant advantage, then using instances of protocol $\pi$ is just as secure as using the ideal functionality $\mathcal{F}$.

From the perspective of the players $P_{i}, \mathcal{F}$ provides a set of subroutine calls that calculate a given function. For instance the subroutine call of the ideal functionality of a PAKE protocol $\mathcal{F}_{\text {pwKE }}$ returns a session key.

The definition of the algorithm of the ITM $\mathcal{F}$ makes sure that sensitive information is hidden from the adversary as long as no "corruption" of parties occurs. Thus, the security targets are inherently guaranteed. With corruptions, we model the case that the adversary gets control over some of the protocol partners and, e.g., is able to retrieve data from that partner's internal memory. In the literature different types of corruption could be distinguished. In so-called "static corruptions" the adversary may gain control only just before starting with an actual protocol execution. In the so-called adaptive or Byzantine corruption models we give the attacker more power by allowing him to corrupt parties at any time during protocol execution. In this paper we consider the stronger Byzantine corruption. 
The original UC theorem from [Can01] allows to analyze the security of a system viewed as a single unit, but it does not guarantee anything if different protocols share some amount of state and randomness, such as, e.g., a hash function functionality. For this reason for our application, the UC theorem cannot be used as-is. Our analysis, just as the strongly related work in [ACCP08] is thus implemented in the framework of universal composition with joint state [CR03].

\subsection{Advantages and drawbacks of security proofs within the UC frame- work}

When comparing the UC framework with alternative approaches, notably the game-based methodology a number of conceptional differences could be distinguished.

One of the most important advantages is that in the $\mathrm{UC}$ framework it is possible to follow a modular approach where more complex solutions could be composed of UC secure submodules by use of the composition theorem [Can00]. This avoids the need for detailed reduction-proofs of composed systems. This fact is strongly linked to the special role of the session id sid individually identifying one of possibly many copies of the ideal functionalities. Within the UC framework, the session id needs to be established prior to entering the protocol.

Note that depending on the actual publication, the session id generation is treated with more or less rigor. In this paper we consider this complexity and include the corresponding two additional messages for sid establishment in our protocol specification. In other work, this aspect seems to be treated with somewhat less rigor.

It is worth noting, that the integration of Diffie-Hellman in composable frameworks typically generates technical challenges [KR17], specifically when considering Byzantine adversaries. It is often infeasible for the simulator to provide the adversary secret scalars being consistent with the previously published group elements. Many previous security proofs were, thus, forced to restrict the analysis to the weaker static adversary model (e.g., [GMR06]). We consider it to be a somewhat exceptional fact that in our proof we were able to circumvent this challenge.

Finally it is worth noting that in the UC framework, the advantage of composability often comes at the expense of somewhat less quantitative formulas regarding computational complexity bounds. Commonly in the UC approach probabilities of algorithmic substeps are qualitatively assumed to be either negligible or not negligible. On this basis it is derived that the real-world protocol could be distinguished from the ideal functionality with negligible or non-negligible probability, often without giving explicit formulas regarding the exact bounds regarding the individual complexity assumptions. It is worth noting that there exist more elaborate composable frameworks considering this aspect with more rigor, see, e.g., [KR17]. This aspect, however, is out of the scope of this paper.

\subsection{Overall proof strategy used in this paper}

In this paper we try to avoid the introduction of new security notions and new ideal functionalities where possible. We first prove that the balanced sub-protocol CPace securely implements the ideal functionality $\mathcal{F}_{\mathrm{pwKE}}$ from $\left[\mathrm{CHK}^{+} 05\right]$ (repeated for reference in figure 6).

We then show by using the UC composition theorem that the combination of the sub-protocols securely implements $\mathcal{F}_{\text {apwKE }}$ from [GMR06] and thus provides conventional "full" augmentation.

We then show that when the ephemeral Diffie-Hellman step within AuCPace is replaced by a long-term key variant, the computational complexity for the server is essentially halved, while preserving the most important guarantee. We will present the corresponding 
idealized functionality $\mathcal{F}_{\text {papwKE }}$ together with the security proof for partially augmented AuCPace.

\section{The AuCPace protocol}

\subsection{Design rationales for the AuCPace protocol}

Our dominating guideline for the protocol development was server-side efficiency. In a typical use case of a remote human-machine interface (HMI) for a resource-constrained target, the HMI client-side has much more powerful computing capabilities.

We assessed power consumption to be one major issue in line with the results of [HL17]. Note that this goes beyond just minimizing the number of exponentiations. Also the choice of a suitable curve, and the need for point compression and point verification sub-steps should be considered. We observed, e.g., that $x$-coordinate-only Diffie-Hellman implementations could provide significant advantages, regarding all of speed, ease-ofimplementation and memory-consumption. We therefore searched for constructions not requiring full group operations, because this could inherently avoid the need for point compression and verification. Specifically on curves with twist security this could allow for more efficient methods against small-subgroup[LL97] attacks.

We also tried to minimize the number of required primitives in the protocols. E.g. we aimed at avoiding the digital signature scheme needed for some constructions, such as the -Method from Gentry, MacKenzie and Ramzan [GMR06].

For AuCPace, we also aimed at aggressively minimizing memory requirements. E.g., it was considered highly desirable, to try to avoid storage for full communication transcripts. Actually as a side-effect of the proof strategy used for AuCPace we don't execute many protocol substeps in parallel and, thus don't require large memory buffers for temporaries and state. Throughout the calculation, we aimed also at minimizing the memory requirement for temporary variables. E.g. for calculating scalar multiplications on elliptic curves we searched for efficient strategies not requiring large pre-computed tables, as e.g. required for window-based speedup techniques. We assumed also that often the ROM limitation might not allow for the tables typically needed for fixed-base point speedups.

\subsection{Parameters of the AuCPace protocol}

The AuCPace protocol is depicted in figures 3 and 4. The protocol is parametrized by

- A password based key derivation function $\mathrm{PBKDF}_{\sigma}$ that is itself parametrized by algorithm settings $\sigma$, specifying e.g. the memory consumption for the password hash or an iteration count. $\mathrm{PBKDF}_{\sigma}$ calculates a string from the password $p w$, a username and a so-called "salt" value. For our reference implementation of AuCPace, we use the memory-hard scrypt [PJ12] password hash parametrized for a memory consumption of 32 MByte.

- A (hyper-)elliptic curve $\mathcal{C}$ with a group $\mathcal{J}$ with co-factor $c_{\mathcal{J}}$ and a Diffie-Hellman $(\mathrm{DH})$ protocol operating on both, $\mathcal{C}$ and its quadratic twist $\mathcal{C}^{\prime}$. We denote the $\mathrm{DH}$ base point in $\mathcal{J}$ with $B$. We don't require full group structure in $\mathcal{J}$ but could also instantiate AuCPace if only group operations modulo negation are available. This can result in more efficient implementations. (For more details regarding this efficiency aspect see the corresponding discussion for the qDSA/EdDSA signature schemes in [RS17].). For our reference implementation, we use Curve25519 [Ber06] and the $x$-coordinate-only Diffie-Hellman protocol X25519. In this paper we follow the recommendation in [Ber14] and reserve the name Curve25519 for the curve and X25519 for the protocol. For the DH protocol we mostly use a simple exponentiation 
notation, even if additional co-factor handling and clamping might apply for the scalars for guaranteeing that the result of any exponentiation is always in $\mathcal{J}$. (The exception from this rule is that we decided explicitly detail the co-factor $c_{\mathcal{J}}$ complexity in figures 3 and 4 because this handling is actually crucial for security.)

- An encoding that represents either a point $Y$ on $\mathcal{J}$ or on the quadratic twist in a fixed-size bit stream. In our reference implementation we make use of an encoding of the $x$-coordinate of the point on Curve 25519 .

- A verification algorithm that checks whether the order of an encoded element $Y$ within $\mathcal{C}$ or $\mathcal{C}^{\prime}$ is large enough for the security target specified by the complexity of the computational Diffie-Hellman problem $(\mathrm{CDH})$ for security parameter $k$. In our reference implementation we make use of Curve25519's twist security and the integrated co-factor of 8 for X25519 scalars, i.e., we just verify that X25519 $(x, Y) \neq 0$.

- A Map2Point operation and its inverse map Map2Point ${ }^{-1}$. Map2Point $(s)$ is required to map a string $s$ to a point from a cryptographically large subgroup $\mathcal{J}_{m}$ of $\mathcal{C}$, such that the discrete logarithm of the point is unknown. The inverse map $s=$ Map2Point ${ }^{-1}(X, l)$ is required to map a point $X \in \mathcal{J}_{m}$ to a bit string $s$ of length 1 bits such that for any randomly sampled $X \in \mathcal{J}_{m}$ the string $s$ is indistinguishable from a random bit string of length $l$. For our reference implementation we use Elligator2 introduced by Bernstein, Hamburg, Krasnova and Lange in [BHKL13] on Curve25519, where the sign of the inverse map result is chosen at random.

- Hash functions $\mathrm{H}_{0} \ldots \mathrm{H}_{4}$. For our reference implementation we use SHA512 where the hash function index is prepended as little-endian four-byte word. (We chose SHA512 for having a high security margin. Imperfections of the hash function for proofs using the random-oracle model, should be considered to be particularly critical.)

We will refer to our reference implementation of AuCPace using the actual choices above as AuCPace25519. While denying any legal responsibility, the authors declare that they are not aware of any intellectual property right or patent limiting the use of AuCPace25519.

\subsection{Configuring the password verifier on the server}

Two basic sub-protocols could be distinguished. In a first sub-protocol the server is given a password-verifier $W=B^{w}$ for storage in its database. This protocol is depicted in figure 3. The second sub-protocol, shown in figure 4, uses the available password verifier for establishing a session key.

The configuration of password verifiers requires one message. We assume, that the specific group $\mathcal{J}$ and the permissible set of PBKDF parametrizations $\sigma$ of the server are known to the client. The client chooses a fresh "salt" value and hashes the password $p w$ to yield a secret scalar $w$. Then a password verifier $W=B^{w}$ is calculated and sent to the server for storage in the database. Optionally user authorization data (uad) is also transmitted. The server then checks whether the parametrization $\sigma$ and the authorization setting to attribute to the user are acceptable and stores the verifier $W$ in the database together with the salt and the authorization settings.

Note that all of the protocols in this paper choose ephemeral fresh "salt" values upon password changes. In some settings, though, we see reasons for using a fixed "pepper" that is chosen once and for all users and servers of a specific plant. When using an ephemeral salt value, we could not avoid some leakage of information. Specifically, with ephemeral "salts" it could not completely be hidden whether or not a user entry is available in the server's database and that a user has changed his password. On the other hand, 
Store password operation for AuCPace

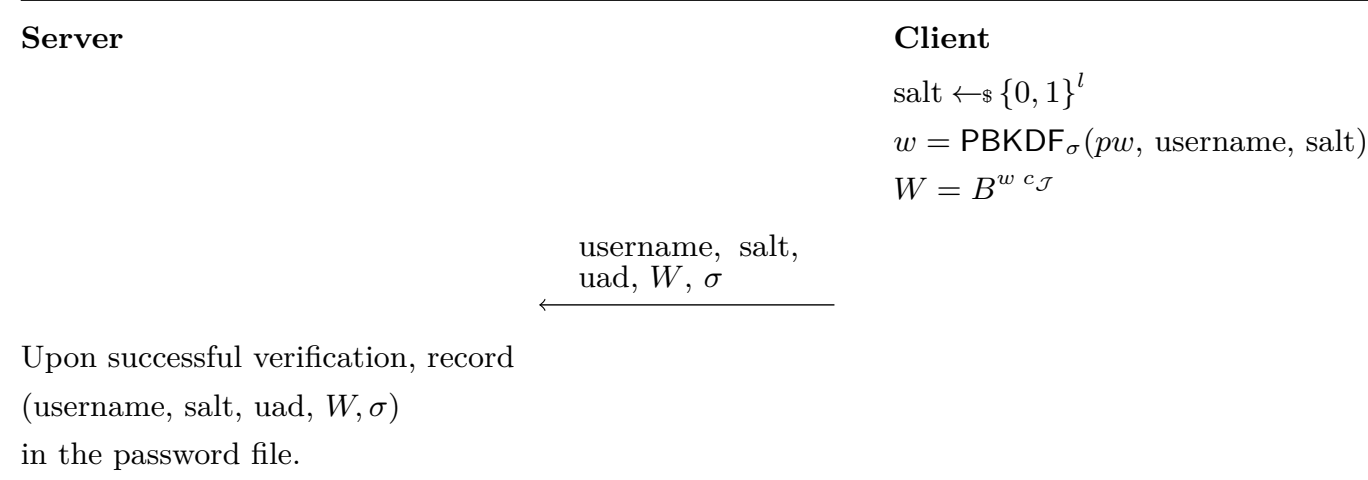

Figure 3: Protocol for password configuration. The optional data element uad represents application data associated with this specific user account, e.g. specifying the granted authorization level on the server.

using a fixed "pepper" known to many parties has the disadvantage that an attacker can pre-compute so-called rainbow-tables.

In line with other V-PAKE papers $\left(\left[\mathrm{PW} 17, \mathrm{CHK}^{+} 05\right.\right.$, JKX18]) for the scope of this paper we concentrate on the security proof for the session establishment only, i.e., for this first sub-protocol we assume that communication is using a confidential channel. We also assume that the client is properly authenticated and has sufficient privileges. More formally, we do not consider adversaries $\mathcal{A}$ that read or modify messages of this sub-protocol.

It is important to note that the password is never passed in clear-text to the server. This also implies that the computationally complex PBKDF function is calculated on the client. Note also that by letting the client choose the "salt" value, we provide a path for distributing password verifiers from a centralized user credential server by use of an offline ticket mechanism, i.e., using a single unidirectional message.

With respect to the generation of the password verifier $W$ our protocol shares some similarities with AugPake [SKI10] and VTBPEKE [PW17] which also use a group element $W=B^{\xi}$ as verifier where a password-derived key $\xi$ is used as a secret exponent.

\subsection{Establishing session keys based on the password $p w$ and the pass- word verifier $W$}

Establishment of the session key $s k$ is realized by a sequence of several steps shown in figure 4. First a subsession id ssid is established as required by the UC framework prior to entering the protocol. (An alternative option would have been to obtain ssid just by concatenating the two random nonces $s$ and $t$. [BLR04])

Secondly, a password related string $P R S$ is calculated. We refer to this sub-protocol as the AuCPace augmentation layer. Establishing PRS involves one message round. After learning the user name, the server fetches the "salt" value from the database. In case that no entry is available for the user name, we suggest to hash a server-specific secret constant together with the username in order to yield a dummy salt value.

The server then transmits its "salt" string together with the value $\sigma$ required for parameterizing the password hash $\mathrm{PBKDF}_{\sigma}$ and an ephemeral public key $P=B^{p c_{\mathcal{J}}}$ and the group representation $\mathcal{J}$ to use for the Diffie-Hellman protocol. Both, server and client entity then calculate a password-derived string $P R S$. While the server uses the password verifier $W$ from its database, the client has to calculate $\mathrm{PBKDF}_{\sigma}$. We neither expect to have permanent storage available on the client, nor do we recommend any permanent caching of "w". If no entry is available for $W$ in the server's password file, or if the 
AuCPace

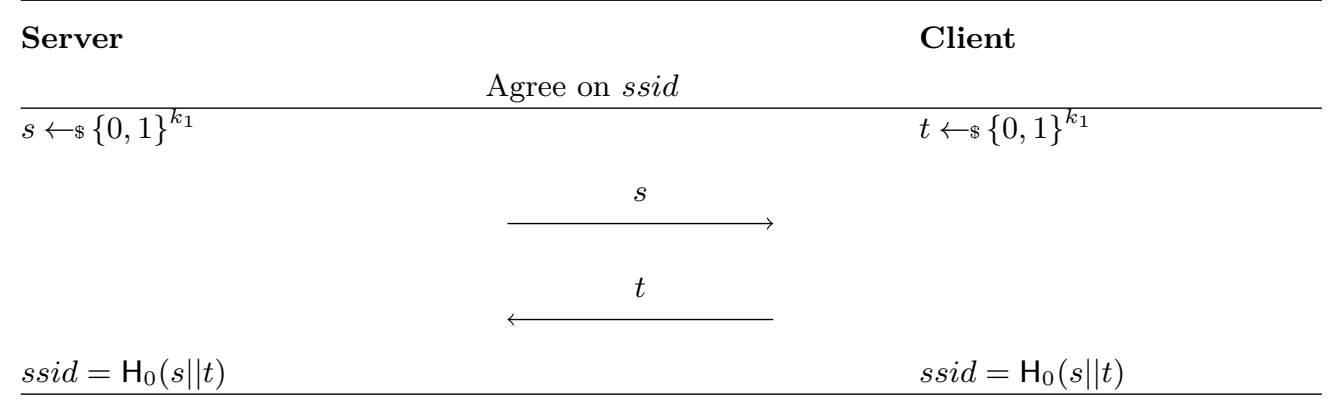

AuCPace Augmentation layer

\begin{tabular}{lc}
\hline$x \leftarrow \$\left\{1 \ldots m_{\mathcal{J}}\right\}$ & \\
$X=B^{x c_{\mathcal{J}}}$ & username \\
& $\longleftarrow$,salt = lookupW(user) \\
& $\stackrel{\mathcal{J}, X, \text { salt }, \sigma}{\longrightarrow}$
\end{tabular}

if lookup failed $P R S \leftarrow \$\{0,1\}^{k_{2}}$, $w=\operatorname{PBKDF}_{\sigma}(p w$, user, salt $)$

else $P R S=W^{x c_{\mathcal{J}}}$ abort if $X$ invalid $P R S=X^{w c_{\mathcal{J}}}$

\begin{tabular}{|c|c|c|}
\hline \multicolumn{3}{|c|}{ CPace substep } \\
\hline$g^{\prime}=\mathrm{H}_{1}($ ssid $\|P R S\| C I)$ & & $g^{\prime}=\mathrm{H}_{1}($ ssid $\|P R S\| C I)$ \\
\hline$G=\operatorname{Map} 2 \operatorname{Point}\left(g^{\prime}\right)$ & & $G=\operatorname{Map} 2 \operatorname{Point}\left(g^{\prime}\right)$ \\
\hline$y_{a} \leftarrow \$\left\{1 \ldots m_{\mathcal{J}}\right\}$ & & $y_{b} \leftarrow \$\left\{1 \ldots m_{\mathcal{J}}\right\}$ \\
\hline$Y_{a}=G^{y_{a} c_{\mathcal{J}}}$ & & $Y_{b}=G^{y_{b} c_{\mathcal{J}}}$ \\
\hline \multicolumn{3}{|c|}{$Y_{a}$} \\
\hline \multicolumn{3}{|c|}{$Y_{b}$} \\
\hline$K=Y_{b}^{y_{a} c_{\mathcal{J}}}$ & & $K=Y_{a}^{y_{b} c_{\mathcal{J}}}$ \\
\hline abort if $Y_{b}$ invalid & & abort if $Y_{a}$ invalid \\
\hline$s k_{1}=\mathrm{H}_{2}($ ssid $\| K)$ & & $s k_{1}=\mathrm{H}_{2}(s s i d \| K)$ \\
\hline \multicolumn{3}{|c|}{ Explicit mutual authentication } \\
\hline$\left.\overline{T_{a}=\mathrm{H}_{3}(s s i d \|}|| s k_{1}\right)$ & & $T_{a}=\mathrm{H}_{3}\left(s s i d \| s k_{1}\right)$ \\
\hline$T_{b}=\mathrm{H}_{4}\left(s s i d \| s k_{1}\right)$ & & $T_{b}=\mathrm{H}_{4}\left(s s i d \| s k_{1}\right)$ \\
\hline \multicolumn{3}{|c|}{$T_{b}$} \\
\hline \multicolumn{3}{|c|}{$T_{a}$} \\
\hline verify $T_{b}$ & & verify $T_{a}$ \\
\hline$s k=\mathrm{H}_{5}\left(s s i d \| s k_{1}\right)$ & & $s k=\mathrm{H}_{5}\left(s s i d \| s k_{1}\right)$ \\
\hline
\end{tabular}

Figure 4: Protocol AuCPace. 
point verification on the client fails, the protocol is continued with a randomly sampled $P R S$ string instead of aborting. Our approach somewhat mitigates the fact that the openly communicated "salt" value leaks some information on the server's password file contents. (At the same time we have to accept more workload when facing some types of denial-of-service attacks.)

Then client and server enter the balanced sub-protocol CPace with the password-derived string $P R S$ as password. There, first an ephemeral generator $G$ is calculated by use of the Map2Point algorithm.

Calculation of $G$ involves a "channel identifier" CI which is hashed together with the $P R S$. In the context of TCP/IP, the CI might be constructed by concatenating unique representations of the server's and client's IP address and TCP port numbers. Hashing CI into $G$ allows us to fend off certain types of relay attacks.

Incorporating the ssid into the calculation guarantees the generator $G$ to be ephemeral also in the partially augmented setting from section 6.1. Note that prefixing the session id is also suggested in section 10.3 of [KTR13] for a security analysis in the IITM model [KTR13] in case that one global random oracle is to be used for an unlimited numbers of subsessions.

After determining $G$ the two parties implement a Diffie-Hellman protocol by exchanging group elements $Y_{a}$ and $Y_{b}$ and deriving a shared secret point $K$. Note that it is mandatory for the receiving party to verify the points $Y_{a}$ and $Y_{b}$. Then a first session key $s k_{1}$ is derived from $K$.

As last sub-protocol, optionally explicit authentication is added by exchange of two authenticator messages $T_{a}$ and $T_{b}$. Finally the session key $s k_{1}$ is refreshed to yield the final session key $s k$.

With respect to the mandatory point verification, we do not impose the conventional requirement that the implementation has to verify that the points $X, Y_{a}$ and $Y_{b}$ are $\in \mathcal{J}$. Instead we impose a less strict requirement that could be implemented more efficiently on some curves, notably if they have secure twists: We require the verification that the order of the respective points is large with respect the required complexity assumption for the $\mathrm{CDH}$ problem, such as to prevent collisions in the resulting points $K$ and $X^{w}$ due to small subgroup attacks.

\subsection{Key difference between AuCPace and previously known SPEKE- based constructions}

The main difference between SPEKE and the balanced CPace subprotocol shown here lies in the fact that CPace works with an ephemeral generator that depends on the session id and the channel identifier CI. This allows for both, a natural way for proving security in the UC framework and a memory-optimized approach for implicitly authenticating data such as the CI.

The feature of the ephemeral generator is shared between CPace and PACE. Unlike PACE, CPace does not need an additional block cipher most certainly employed for patent circumvention only. Note that this reduces both, implementation complexity and the number of assumptions required for the proof. For CPace there is no need for the ideal-cipher assumption.

Most importantly, AuCPace allows for two different levels of augmentation. If an ephemeral key pair $(x, X)$ is used, we obtain the conventional security guarantees of an augmented PAKE protocol. Additionally, AuCPace allows for a "partially augmented" mode where the key pair $(x, X)$ is of long-term type and generated once during password registration. The security guarantee of partial PAKE augmentation will be formally introduced in section 6 . For this reason we will continue the more detailed comparison between AuCPace and other protocols only in section 7. We invite readers wishing to 
The protocol CPace is parametrized by a security parameter $k$. CPace implements the hashes with output size $2 k \mathrm{H}_{1}$ and $\mathrm{H}_{2}$ by the functionality $\mathcal{F}_{\mathrm{RO}}$. The protocol also uses a DiffieHellman key exchange protocol (written in exponentiation notation $X^{y}$ ) operating on points $X$ on a group modulo negation $\mathcal{J}$ of order $m_{\mathcal{J}}$ and its twist. It also uses a Map2Point primitive mapping a string of length $2 k$ on an element $Y$ where $Y^{r}$ generates $\mathcal{J}$. It finally uses a $\operatorname{Verify}(Y)$ algorithm checking for the subgroup order of a given $Y$ on either $\mathcal{J}$ or the twist.

Protocol:

1. When $P_{j}$ receives input (NewSession, sid, $P_{i}, P_{j}, p w$, responder role), he calculates $g^{\prime}=$ $\mathrm{H}_{1}\left(\operatorname{sid}\left\|P_{i}\right\| P_{j} \| p w\right)$ and $G=$ Map2Point $\left(g^{\prime}\right)$. He then samples a fresh nonzero scalar $y_{b}$ with $0<y_{b}<m_{\mathcal{J}}$ and calculates $Y_{b}=G^{y_{b}}$. He sets up a session record (sid, $P_{i}, y_{b}, Y_{b}$, responder role) and marks it as fresh. He then waits for a (CPace initiator, sid, $P_{i}, Y_{a}$ ) message.

2. When $P_{i}$ receives input (NewSession, sid, $P_{i}, P_{j}, p w$, initiator role), he calculates $g^{\prime}=$ $\mathrm{H}_{1}\left(\right.$ sid $\left.\left\|P_{i}\right\| P_{j} \| p w\right)$ and $G=\operatorname{Map} 2 \operatorname{Point}\left(g^{\prime}\right)$. He then samples a fresh nonzero scalar $y_{a}$ with $0<y_{a}<m_{\mathcal{J}}$ and calculates $Y_{a}=G^{y_{a}}$. He sets up a session record (sid, $P_{j}, y_{a}$, initiator role) and marks it as fresh. He then sends (CPace initiator, sid, $P_{i}, Y_{a}$ ) to $P_{j}$.

3. When $P_{j}$ receives input (CPace initiator, sid, $P_{i}, Y_{a}$ ) and finds a fresh session record ( $s i d, P_{i}, y_{b}, Y_{b}$, responder role) it sends message (CPace responder, sid, $P_{j}, Y_{b}$ ) message to $P_{i}$. He then calculates $\operatorname{Verify}\left(Y_{a}\right)$. If the point order is sufficient for security parameter $2 k$ it calculates $K=Y_{a}^{y_{b}}$ and $s k=\mathrm{H}_{2}(s i d \| K)$ and outputs (sid, sk). It aborts otherwise. In either case it marks the session record as completed.

4. When $P_{i}$ receives input (CPace responder, sid, $\left.P_{j}, Y_{b}\right)$ and finds a fresh session record (sid, $P_{j}, y_{a}$, initiator role) he calculates $\operatorname{Verify}\left(Y_{b}\right)$. If the point order is sufficient for security parameter $2 k$ it calculates $K=Y_{b}^{y_{a}}$ and $s k=\mathrm{H}_{2}($ sid $\| K)$ and outputs (sid, sk). It aborts otherwise. In either case it marks the session record as completed.

Figure 5: CPace Protocol definition for the proof of indistinguishability.

directly continue with this discussion to skip the rather technical proof sections 4 to 6 for a first reading.

\section{Proof of indistinguishability for the balanced sub-protocol CPace}

In this section we will deal with the balanced PAKE protocol CPace corresponding to the middle part of figure 4. We formalize this sub-protocol in figure 5.

In this proof we show that the protocol in figure 5 emulates the functionality $\mathcal{F}_{\text {pwKE }}$ (figure 6) from $\left[\mathrm{CHK}^{+} 05\right] . \mathcal{F}_{\mathrm{pwKE}}$ receives the passwords $p w$ from the environment-controlled parties $P_{i}$ and $P_{j}$ and returns upon a NewKey request the same random session key $s k$ if and only if (iff) the passwords match.

In $\left[\mathrm{CHK}^{+} 05\right] \mathcal{F}_{\text {pwKe }}$ has been used in the context of static adversaries only. Here we observed the need to more clearly specify the behavior of $\mathcal{F}_{\text {pwKE }}$ in case of adaptive corruptions. As in the static corruption model used in $\left[\mathrm{CHK}^{+} 05\right]$, we let $\mathcal{F}_{\text {pwKE }}$ give party $P_{i}$ 's password to $\mathcal{S}$ upon corruption. In addition, we need to handle the case that $\mathcal{S}$ corrupts a party $P_{j}$ after a session key $s k$ has been already sent to party $P_{i}$. If $P_{i}$ and $P_{j}$ use the same password $p w$, we send $\mathcal{S}$ the honest party's session key $s k$, otherwise (different passwords) we send $\mathcal{S}$ a randomly sampled key $s k^{\prime}$. This is required for giving $\mathcal{S}$ the possibility to continue behaving as a honest party.

For the security analysis in this section we need to map the inputs and outputs of the protocol from figure 4 to the notation used for defining the ideal functionality $\mathcal{F}_{\text {pwKE }}$ in 
The functionality $\mathcal{F}_{\text {pwKE }}$ is parametrized by a security parameter $k$. It interacts with an adversary $\mathcal{S}$ and a set of parties via the following queries:

Upon receiving a query (NewSession, sid, $P_{i}, P_{j}, p w$, role) from party $P_{i}$ :

Send (NewSession, sid, $P_{i}, P_{j}$,role) to $\mathcal{S}$. In addition, if this is the first NewSession query, or if this is the second NewSession query and there is a record $\left(P_{j}, P_{i}, p w^{\prime}\right)$, then record $\left(P_{i}, P_{j}, p w\right)$ and mark this record fresh.

Upon receiving a query (TestPwd, sid, $\left.P_{i}, p w^{\prime}\right)$ from the adversary $\mathrm{S}$ :

If there is a record of the form $\left(P_{i}, P_{j}, p w\right)$ which is fresh, then do: If $p w=p w^{\prime}$, mark the record compromised and reply to $\mathcal{S}$ with "correct guess". If $p w \neq p w^{\prime}$, mark the record interrupted and reply with "wrong guess".

Upon receiving a query (NewKey, $s i d, P_{i}, s k$ ) from $\mathcal{S}$ where $|s k|=k$ : If there is a record of the form $\left(P_{i}, P_{j}, p w\right)$, and this is the first NewKey query for $P_{i}$, then:

- If this record is compromised, or either $P_{i}$ or $P_{j}$ is corrupted, then output ( $s i d, s k$ ) to player $P_{i}$.

- If this record is fresh, and there is a record $\left(P_{j}, P_{i}, p w^{\prime}\right)$ with $p w^{\prime}=p w$, and a key $s k^{\prime}$ was sent to $P_{j}$ and $\left(P_{j}, P_{i}, p w\right)$ was fresh at the time, then output $\left(s i d, s k^{\prime}\right)$ to $P_{i}$.

- In any other case, pick a new random key $s k^{\prime}$ of length $k$ and send $\left(s i d, s k^{\prime}\right)$ to $P_{i}$.

Either way, mark the record $\left(P_{i}, P_{j}, p w\right)$ as completed.

Figure 6: Ideal functionality $\mathcal{F}_{\text {pwKE }}$ from $\left[\mathrm{CHK}^{+} 05\right]$ re-presenting balanced PAKE without explicit authentication.

figure 6. The password related components ( $W^{x}$ and $X^{w}$ respectively) correspond to the passwords $p w$ and the resulting session key $s k$ from figure 6 corresponds to $s k 1$ in figure 4 . We define the channel identifier $C I$ to be the concatenation of $P_{i}$ and $P_{j}$.

\subsection{Proof strategy}

Our proof closely follows the strategy from [ACCP08]. We also use a sequence of games $\mathrm{G}_{0}$ to $\mathrm{G}_{4}$ in which the simulator algorithms $\mathcal{S}_{0}$ to $\mathcal{S}_{4}$ are executed. We organize these algorithms $\mathcal{S}_{n}$ as a combination of independent ITI that only interact through their welldefined APIs and have internal state (tape) that is not accessible from the outside. Initially we have one such ITI for each simulated honest party $P_{i}$, the hash functionality $\mathcal{F}_{\mathrm{RO}}$ and one ITI executing the algorithm of the real-world adversary $\mathcal{A}$. We let $\mathcal{S}_{n}$ invoke the other ITI during the course of the execution, i.e., we treat the real-world adversary algorithm $\mathcal{A}$ as a black box subroutine library for $\mathcal{S}_{n}$.

When $\mathcal{A}$ decides to corrupt a party $P_{i}$, we need to provide it all of the corrupted ITI's internal accessible state. The subsequent behavior of this party is then controlled by $\mathcal{A}$. The adversary is also given the secret scalars used in the real-world protocol. Finally, we give $\mathcal{S}_{n}$ also access to an ITM $\mathcal{F}_{n}$ in each game, where $\mathcal{F}_{0}$ is initially not providing any service. In each game we extend the functionality of $\mathcal{F}_{n}$ until it implements $\mathcal{F}_{\text {pwKE}}$.

In the games in our proof we re-factor the algorithms $\mathcal{S}_{n}$ such that each change is indistinguishable for the environment $\mathcal{Z}$.

At the end of the game sequence, we end up with an algorithm $\mathcal{S}_{4}$ that makes only calls to $\mathcal{F}_{4}$ which itself implements exactly the ideal functionality $\mathcal{F}_{\text {pwKE }}$. 


\subsection{Game-based proof}

Theorem 1. The protocol CPace from figure 5 securely realizes $\mathcal{F}_{p w K E}$ in the $\mathcal{F}_{R O}$ hybrid model in the presence of adaptive adversaries under the assumptions

- of the hardness of the computational Diffie-Hellman problem in $\mathcal{J}$ (AO)

- that for any given randomly sampled group element $X \in \mathcal{J}_{m}$ the inverse map $\operatorname{Map2Point}^{-1}(X, l)$ is indistinguishable from a random string of length l (A1)

- that for any base point $B$ and random string s the probability for finding an exponent $y$ such that $B^{y}=$ Map2Point $(s)$ is negligible (A2).

Note that the assumption A1 is covered by the security guarantees of maps such as Elligator2 [BHKL13] and will allow us for covering the case of fully adaptive adversaries. Without this assumption we would not be able to resolve the commitment problem linked to the Diffie-Hellman substep for adaptive adversaries. (Note that without A1 a proof considering only static corruption still remains possible.). Assumption A2 matches the requirements from [BFK09] for the Map2Point operation in PACE.

\subsubsection{Game $\mathrm{G}_{0}$ : Real Game}

$\mathrm{G}_{0}$ is the real game in the random-oracle model using the functionality $\mathcal{F}_{\mathrm{RO}}$ from figure 7 . The parties $P_{i}$ receive NewSession queries from all simulated honest parties. These queries contain the passwords provided by the environment $\mathcal{Z} . P_{i}$ then executes the actions of initially honest parties in the protocol. In the event of corruptions, the internal state of the parties is passed to the real-world adversary algorithm $\mathcal{A}$. The subroutine library $\mathcal{F}_{0}$ is empty.

\subsubsection{Game $G_{1}$ : Simulation of the random oracle}

Here we modify the previous game by replacing the calls $\mathrm{H}_{n}(q)$ to the original $\mathcal{F}_{\mathrm{RO}}$ hash ITI by a separate implementation. We let $\mathcal{S}_{1}$ maintain an initially empty list $\Lambda$ of value pairs $(n, q, g, r)$. For any hash query $\mathrm{H}_{n}(q)$ such that $(n, q, *, r)$ appears in $\Lambda$ from any of the ITI libraries, the returned answer is $r$. In case that no query $q$ has yet occurred, we handle separately the cases of $n=1$ and $n \neq 1$. In case of $n \neq 1$ we implement the conventional random-oracle model by choosing a new random $r$ of length $k$, by storing $(n, q, 0, r)$ in $\Lambda$ and by returning $r$ to the calling ITI.

For $n=1$ instead, we aim at generating a random string $r$ such that the discrete logarithm of the point Map2Point $(r)$ is known. For this purpose we first generate a random point $G$ whose discrete logarithm is known and use the inverse map Map2Point ${ }^{-1}(G, k)$ for converting it into a bit string of length $k$. We use the guarantee, that for any random point $G$ the string $r=$ Map2Point $^{-1}(G, k)$ is indistinguishable from a random value.

For calculating the random point $G$, we first choose a random nonzero value $g$ being smaller than the order of the group. We calculate the point on $\mathcal{J}, G=B^{g}$. We then test whether $G$ is in the image of Map2Point, $\mathcal{J}_{m}$. If $G$ is not in the image, we restart with a new random value $g$ until $G \in \mathcal{J}_{m}$. This is guaranteed to succeed in probabilistic polynomial time because $\mathcal{J}_{m}$ is large by assumption A2.

Then we calculate $r=$ Map2Point $^{-1}(G, k)$, record $(1, q, g, r)$ in $\Lambda$ and return $r$.

Since the inverse map returns a string indistinguishable from a random string by the assumption $A 1 G_{0}$ and $G_{1}$ are indistinguishable for the environment. 


\subsubsection{Game $\mathrm{G}_{2}$ : Handle the case that an impersonating adversary wins by chance}

Here we handle the case that an impersonating adversary succeeds in calculating the session key $s k$ without querying the hash oracle $\mathrm{H}_{2}$ for $K$. In this case we let $\mathcal{S}_{2}$ abort. This case occurs with negligible probability. $G_{2}$ is, thus, indistinguishable from $G_{1}$.

\subsubsection{Game $\mathrm{G}_{3}$ : Restrict the access to the password.}

Here, when receiving the passwords for party $P_{i}$ from the environment, we let $P_{i}$ pass them directly to the subroutine library $\mathcal{F}_{3}$ and allow the rest of the program $\mathcal{S}_{3}$ no longer access the password unless the simulated party $P_{i}$ gets corrupted. We let $\mathcal{S}_{3}$ inform $\mathcal{F}_{3}$ in case that a party got corrupted such that $\mathcal{F}_{3}$ returns the password in this case. We add also an implementation of the TestPwd query to $\mathcal{F}_{3}$ and implement it according to the spec. of $\mathcal{F}_{\text {pwKE }}$. In this game, we preliminarily also add a SamePwd query to $\mathcal{F}_{3}$ that returns true if the passwords match.

As a result, the password-derived generator $G$ from figure 4 is no longer known to the ITI $P_{i}$. We therefore cannot start the original protocol from the beginning and need to refactor the ITM for the parties as well.

Instead we let the ITI $P_{i}$ calculate the protocol messages $Y_{a}$ and $Y_{b}$ to be random multiples of the group's base point $B, Y_{a}=B^{z_{a}}$ and $Y_{b}=B^{z_{b}}$. We also let $P_{i}$ maintain an initially empty list $\Gamma$ and store the secret scalars of simulated honest parties ( sid, $P_{i}, y$ unknown, $\left.z_{a}\right)$ and $\left(\right.$ sid, $P_{j}, y$ unknown, $\left.z_{b}\right)$ in this list together with the respective session id.

Since powers of $G$ from game $\mathrm{G}_{2}$ generate $\mathcal{J}$, the resulting points $Y_{a}, Y_{b}$ take any value on $\mathcal{J}$ with equal probability (except for the neutral element) for honest parties. So do the points generated in $G_{3}$. The public messages in $G_{2}$ and $G_{3}$ are, thus, indistinguishable for the environment.

As soon as both messages $Y_{a}$ and $Y_{b}$ have been delivered by $\mathcal{A}$ we calculate the Diffie-Hellman results using the received points and the local exponents $z_{a}$ and $z_{b}$. If the respective result points differ, we know that $\mathcal{A}$ has modified the messages in a destructive way and record for this session a flag DHFails.

In case that a party $P_{i}$ gets corrupted before calculating $K$, we need to hand over $\mathcal{A}$ something being consistent with the internal state from $P_{i}$ in $\mathrm{G}_{2}$, notably the values $y$ ( $y_{a}$ or $y_{b}$ respectively for client and server).

In the case of corruption, $\mathcal{F}_{3}$ grants us access to the secretly stored $p w$ from its internal state. $\mathcal{S}_{3}$ may then take the password and the session id and make a corresponding hash query to $\mathrm{H}_{1}$. We then retrieve the secret scalar value $g$ from $\Lambda$. We fetch the party $P_{i}$ 's secret scalar $z$ from $\Gamma$ and calculate $y=z / g$. We add the party's secret scalar $z$ to the record in $\Gamma$ with $y$ and hand over $y, p w$ to $\mathcal{A}$.

In case that any party gets corrupted after calculating $K$ but before calculating the final $\mathrm{H}_{2}$, we perform the secret scalar correction above and recalculate a new $K=Y_{r}^{y}$ by using the received point value $Y_{r}$ and pass $K$ to $\mathcal{A}$.

The code for the verification handling for the received points $Y_{r}$ can remain unchanged in comparison to Game $\mathrm{G}_{2}$.

In case that the point verification fails for any party, we do not generate a session key and do not need to calculate the final hash $\mathrm{H}_{2}($ sid $\| K)$. In case that the final hash $\mathrm{H}_{2}$ needs to be calculated for the first of the parties $P_{i}$ and $P_{i}$ is still honest, we need to provide a session key to $P_{i}$. (Note that this could be either server or client.) We distinguish three cases.

- If the other party was corrupted earlier, we know the other party's password $p w^{\prime}$. We then may issue a TestPwd query to $\mathcal{F}_{3}$. If the guess was correct, we learn the local secret scalar value $y$ by the method described above and calculate $K=Y_{r}^{y}$ with the received remote point $Y_{r}$. We query $s k=\mathrm{H}_{2}($ sid ||$K)$ for the corrected value 
of $K$ and return the result to $P_{i}$. If the guess was wrong, we sample a new random key $s k$ and return it to $P_{i}$.

- If the other party is still honest, we sample a new random key $s k$ and send it to $P_{i}$ and record this session key together with the session id and the party identifiers $\left(\right.$ sid $\left., P_{i}, P_{j}, s k\right)$.

- If the other party is impersonated by $\mathcal{A}$ we also sample a new random key $s k$ and send it to $P_{i}$ and record this session key together with the session id and the party identifiers $\left(s i d, P_{i}, P_{j}, s k\right)$. Note that (according to the previous game), we will be returning a distinguishable key $s k$ iff $\mathcal{A}$ somehow managed to guess the value $K=Y_{r}^{y}$ in $G_{2}$. We will calculate the corresponding probability GuessK in section 4.3.

The remaining task is to calculate the session key $s k$ for a second party $P_{j}$ if it is not corrupted until the very end or corrupted before calculating $s k$. In any of these two cases, we know that two messages $Y_{a}$ and $Y_{b}$ must have been delivered by $\mathcal{A}$, and we, thus, have access to the DHFails marker and that the received points are not from a low order sub-group (or the neutral element). Also, because we know that we have to simulate session key generation for the second time, we know that the first party was honest until the end of the protocol.

If the second party is also honest until the very end, we make a SamePwd query introduced temporarily to $\mathcal{F}_{3}$. If the passwords match and if the session is not marked as DHFails, we return the same $s k$ value to $P_{j}$ as for the first party, otherwise we sample a new random key $s k^{\prime}$ and return this one to $P_{j}$.

In case that the second party $P_{j}$ gets corrupted after calculating $K$ we first correct $K$ using the secret exponent $g$ retrieved from $\Lambda$. In case that we recognized destructive modification of the Diffie-Hellman points by the DHFails marker for the session, we just sample a new value for $s k^{\prime}$ by the interface of the random oracle $s k^{\prime}=\mathrm{H}_{2}(s i d \| K)$ and pass $s k^{\prime}$ to $\mathcal{A}$. There is only a negligible chance of collision with the key $s k$ sent to the first party, since both $s k$ and $s k^{\prime}$ have been randomly sampled. There is also only a negligible chance that $\mathcal{A}$ managed to make both parties issue the same session key despite different passwords by modification of the transmitted points. For this reason $G_{3}$ and $\mathrm{G}_{2}$ are indistinguishable for this case.

If the Diffie-Hellman points have not been modified in a destructive way (DHFails not recognized), the session key issued in $G_{2}$ depends on the password. We learn the party's password $p w$ from $\mathcal{F}_{3}$. We then may issue a TestPwd query to $\mathcal{F}_{3}$. If the guess was correct, we have to provide the same session key to $\mathcal{A}$ as for the first party if DHFails is not recognized. For this purpose, we program the value $\mathrm{H}_{2}(s i d \| K):=s k$ to the session key returned to the first party. This could fail only, if the oracle $\mathrm{H}_{2}$ already has been queried for $(\operatorname{sid} \| K)$, again corresponding to the probability GuessK that we deal with in section 4.3. (Note that it is for this re-programming operation that we will later need to be granted access to the session key issued to the client by the ideal functionality $\mathcal{F}_{\text {pwKE }}$. Otherwise we could not give $\mathcal{S}$ access to the session key that would have been calculated by honest parties for corruptions occurring just after executing the hash function.)

The messages $Y_{a}, Y_{b}$ generated in Game $\mathrm{G}_{2}$ and $\mathrm{G}_{3}$ are indistinguishable for the environment because they come from the same distribution. (Note that for this precise aspect, the appropriate co-factor handling was mandatory!) Also the session keys are sampled from an indistinguishable uniform distribution in both cases. Session keys delivered to parties $P_{i}$ and $P_{j}$ match under the same conditions as in $\mathrm{G}_{2}$. Inserting points on the group's twist by the adversary always leads to different session keys for both parties, just as in $G_{2} \cdot G_{2}$ and $G_{3}$ are, thus, indistinguishable for $\mathcal{Z}$. 


\subsubsection{Game $\mathrm{G}_{4}$ : Merge the key generation procedures to the functionality $\mathcal{F}_{4}$.}

In this game we essentially only do code-refactoring and move the code responsible for session key generation to the ITM $\mathcal{F}_{4}$. We make $\mathcal{F}_{4}$ implement exactly the functionality $\mathcal{F}_{\text {pwKE. }}$. Note that we need maintain the queries within $\mathcal{F}_{4}$ that give access to the passwords $p w$ in case of corruptions. We also need to add a query returning the session key delivered to the client in case of late adaptive corruptions of the second party (server), as discussed above. We remove the SamePwd query from the list of queries for $\mathcal{F}_{4}$ because now, $\mathcal{F}_{4}$ could easily check itself for password identity in its internal storage. Within $\mathcal{S}_{4}$ we finally replace the sampling of the session keys by calls to the NewKey query of the ideal functionality.

Since between $G_{3}$ and $G_{4}$ no functionality change is present, $G_{3}$ and $G_{4}$ are indistinguishable for $\mathcal{Z}$.

\subsection{Proof that probability GuessK in $G_{3}$ is negligible}

In $\mathrm{G}_{2}$ an eavesdropping adversary has access to the published points $Y_{a}$ and $Y_{b}$ generated by honest parties. In $\mathrm{G}_{2}$ he could make guesses for the password and derive the corresponding generator $G$ from the known sid. Successfully guessing $K$ for a given password is equivalent to the $\mathrm{CDH}$ problem, and is of negligible probability by assumption A0.

$\mathrm{G}_{3}$ can be distinguished by $\mathcal{Z}$ from $\mathrm{G}_{2}$ iff the impersonating adversary succeeds in obtaining the real-world protocol's honest party point $K=Y_{a}^{y_{b}}$ in $\mathrm{G}_{2}$ without knowing the honest player's password. The reasoning is identical for both, server and client, so we consider that $\mathcal{A}$ impersonates the server.

Note that $Y_{a}$ is under control of the adversary and may be either on the curve or on its twist. We know, however, that irrespective of the curve the order of the point $Y_{a}$ is large, making $\mathrm{CDH}$ untraceable by assumption $\mathrm{A} 0$.

In $\mathrm{G}_{2} \mathcal{A}$ could successfully run guesses on $K$ if he knew the exponent of the generator $G=B^{g}$ for a given password $p w$. For this purpose, $\mathcal{A}$ could transmit $Y_{a}=B^{y_{a}}$ and could exploit the knowledge on $Y_{b}$ by guessing $K=Y_{b}^{1 / g}$. By assumption A2 the probability that $\mathcal{A}$ knows $g$ happens with negligible probability.

In case that both scalars $g$ and $y_{b}$ are unknown to $\mathcal{A}$ the problem of calculating $K=Y_{a}^{y_{b}}$ from known $Y_{b}=B^{g * y_{b}}$ is intracable for any base point $B$. Note that the secret scalar $g$ is known to exist, but known to no party according to assumption A2.

Note that if one more conservatively assumes that the adversary managed to control the generator $G$ somehow, possibly by biasing the sid and by exploiting two simultaneous flaws in both, the hash and the Map2Point primitive, one would obtain the PACE-DH problem from [BFK09]. In the very same paper this one is shown to be as hard as DH in the generic model of Shoup [Sho97] and conjectured to be as hard as CDH.

In any case the probability GuessK is, thus, negligible. This makes the real-world execution of the CPace protocol indistinguishable from the ideal world.

\subsection{Remarks regarding the ordering of the messages and efficiency}

Note that in this proof we have assumed that the server party starts with the communication round. In fact, since the services provided to the two parties by the ideal functionality are identical and since the protocol is perfectly symmetric, we could interchange the server and client roles for the balanced PAKE sub-protocol CPace. This might be useful, specifically in case that the scalar multiplication takes comparable time as message delivery. 
The functionality $\mathcal{F}_{\mathrm{RO}}$ proceeds as follows, running on security parameter $k$ with parties $P_{1}, \ldots, P_{n}$ and an adversary $\mathcal{S}$ :

$\mathcal{F}_{\mathrm{RO}}$ keeps a list $L$ (which is initially empty) of pairs of bit strings.

Upon receiving a value $($ sid, $m)$ with $\left(m \in\{0,1\}^{*}\right)$ from some party $P_{i}$ or from $\mathcal{S}$, do:

- If there is a pair $(m,(\tilde{h}))$ for some $\tilde{h} \in\{0,1\}^{k}$ in the list $L$, set $h:=\tilde{h}$.

- If there is no such pair, choose uniformly $h \in\{0,1\}^{k}$ and store the pair $(m, h) \in L$.

Once $h$ is set, reply to the activating machine (i.e., either $P_{i}$ or $\mathcal{S}$ ) with $($ sid,h).

Figure 7: Ideal functionality $\mathcal{F}_{\mathrm{RO}}$.

\section{Proof for the augmented protocol AuCPace}

\subsection{Technical details}

For carrying out the proof, we aim at re-using functionalities from previous papers wherever possible, specifically the ideal functionality $\mathcal{F}_{\text {apwKE }}$ from [GMR06].

However, for our protocol we could not use it as-is because $\mathcal{F}_{\text {apwKE }}$ aborts in case that the server does not find a password entry in its file. In our protocol, we aim at keeping the information which users have a database entry as confidential as possible. Therefore we continue with a random $P R S$ string instead of aborting, thus concealing the information at least from the low-motivation and low-skill attacker. Note that full confidentiality for database contents could be fully realized only when using a server-specific "pepper". With a transmitted "salt" we also couldn't hide the events of password changes. We first considered re-phrasing $\mathcal{F}_{\text {apwKE }}$ but finally decided to stick with the established functionality which aborts if no password database entry is available (in line with [GMR06, JKX18]).

The second technical aspect to consider is the handling of the $\operatorname{PBKDF}_{\sigma}$ (pw,username,salt) function. In the proof, we treat PBKDF as a separate hash function $\mathrm{H}_{6}$ and model it as a random oracle $\operatorname{PBKDF}_{\sigma}(p w$,username,salt $)=\mathrm{H}_{6}(p w\|\sigma\|$ username $\|$ salt $)$.

The third technical aspect stems from the fact, that the UC simulation model based on Turing machines does not naturally allow for the concept of human users with "user names" and authorizations. Instead we assume that the client's identifier $P_{i}$ takes over the role of the user name and ignore the concept of authorization here. The full protocol as used for the proof is shown in figure 9 .

We adhere to the convention from [GMR06] and use "server compromise" for the event of stealing the server's persisted state. We use the terminology denote "corruption" for events where the adversary gains control over a party during session establishment.

\subsection{Proof strategy for the augmented protocol}

With respect to simulation, we need to distinguish password storage and session establishment. During password storage we proceed as in [GMR06] and do not actually give the adversary $\mathcal{A}$ any power. We allow $\mathcal{A}$ to compromise the server after receiving the message configuring the password. For this reason, here the simulation of message transmission does not provide any difficulty. We let $\mathcal{S}$ just forward the StorePWfile query to the $\mathcal{F}_{\text {apwKe }}$ functionality and send an empty StorePWfileSvr message to the server.

With respect to the session establishment, we again consider fully-adaptive adversaries which are able to read and modify the messages. The most complex part of the proof will be handling of compromise of the server database. Just as for the proof of the balanced sub-protocol CPace, we proceed by using a sequence of games where $\mathrm{G}_{0}$ corresponds to the real world and $G_{4}$ corresponds to the ideal world. In each of these games, we consider 
The functionality $\mathcal{F}_{\text {apwKe }}$ is parametrized by a security parameter $k$. It interacts with an adversary $\mathcal{S}$ and a set of parties via the following queries:

\section{Password storage and authentication sessions}

Upon receiving a query (StorePWfile, sid, $P_{i}, P_{j}, p w$ ) from party $P_{i}$ :

If this is the first StorePWfile query, store password data record (file, $P_{i}, P_{j}, p w$ ) and mark it uncompromised.

Upon receiving a query (CltSession, sid, ssid, $\left.P_{i}, p w\right)$ from party $P_{i}$ :

Send (CltSession, sid, ssid, $\left.P_{j}, P_{j}\right)$ to $\mathcal{S}$, and if this is the first CltSession query for ssid, store session record (ssid, $\left.P_{i}, P_{j}, p w\right)$ and mark it fresh.

Upon receiving a query (SvrSession, sid, ssid) from party $P_{j}$ :

If there is a password data record (file, $P_{i}, P_{j}, p w$ ) then send (SvrSession, sid, ssid, $P_{i}, P_{j}$ ) to $\mathcal{S}$, and if this is the first SvrSession query for ssid, store session record $\left(s s i d, P_{j}, P_{i}, p w^{\prime}\right)$ and mark it fresh.

\section{Stealing password data}

Upon receiving a query (StealPWfile, sid) from adversary $\mathcal{S}$ :

If there is no password data record, reply to $\mathcal{S}$ with "no password file". Otherwise do the following. If the password data record (file, $P_{i}, P_{j}, p w$ ) is marked uncompromised, mark it as compromised. if there is a tuple (offline,$p w^{\prime}$ ) stored with $p w=p w^{\prime}$, send $p w$ to $\mathcal{S}$, otherwise reply to $\mathcal{S}$ with "password file stolen".

Upon receiving a query (OfflineTestPwd, sid, $p w^{\prime}$ ) from adversary $\mathcal{S}$ :

If there is no password data record, or if there is a password record (file, $P_{i}, P_{j}, p w$ ) that is marked uncompromised, then store (offline,$p w^{\prime}$ ). Otherwise, do: If $p w=p w^{\prime}$, reply to $\mathcal{S}$ with "correct guess". If $p w \neq p w^{\prime}$, reply with "wrong guess".

\section{Active session attacks}

Upon receiving a query (TestPwd, sid, ssid, $\left.P, p w^{\prime}\right)$ from adversary $\mathcal{S}$ :

If there is a session record of the form (ssid, $\left.P, P^{\prime}, p w\right)$ which is fresh, then do: If $p w=p w^{\prime}$, mark the record compromised and reply to $\mathcal{S}$ with "correct guess". Otherwise, mark the session record interrupted and reply with "wrong guess".

Upon receiving a query (Svrlmpersonate, sid, ssid) from adversary $\mathcal{S}$ :

If there is a session record of the form $\left(s s i d, P_{i}, P_{j}, p w\right)$ which is fresh, then do: If there is a password data record (file, $P_{i}, P_{j}, p w$ ) that is marked compromised, mark the session record compromised and reply to $\mathcal{S}$ with "correct guess", else mark the the session record interrupted and reply with "wrong guess".

\section{Key Generation and Authentication}

Upon receiving a query (NewKey, sid, ssid, $P$, key) from adversary $\mathcal{S}$, where $|k e y|=k$ : If there is a record of the form $\left(s s i d, P, P^{\prime}, p w\right)$ that is not marked completed, then:

- If this record is compromised, or either $P$ or $P^{\prime}$ is corrupted, then output (sid, ssid, key) to $P$.

- If this record is fresh, there is a session record $\left(s s i d, P^{\prime}, P, p w^{\prime}\right), p w^{\prime}=p w$, a key $k e y^{\prime}$ was sent to $P^{\prime}$, and $\left(s s i d, P^{\prime}, P, p w\right)$ was fresh at the time, then let $k e y^{\prime \prime}=k e y^{\prime}$, else pick a random key $k e y^{\prime \prime}$ of length $k$. Output (sid, ssid, key $\left.y^{\prime \prime}\right)$ to $P$.

- In any other case, pick a random key $k e y^{\prime \prime}$ of length $k$ and output (sid, ssid,key") to P.

Finally, mark the record (ssid, $\left.P, P^{\prime}, p w\right)$ as completed.

Upon receiving a query (TestAbort, sid, ssid, $P$ ) from adversary $\mathcal{S}$ :

If there is a session record of the form (ssid, $\left.P, P^{\prime}, p w\right)$ that is not marked completed, then:

- If this record is fresh, there is a record $\left(\operatorname{ssid}, P^{\prime}, P, p w^{\prime}\right)$, and $p w^{\prime}=p w$, let $b^{\prime}=$ succ.

- In any other case let $b^{\prime}=$ fail

Send $b^{\prime}$ to $\mathcal{S}$. If $b^{\prime}=$ fail, send (abort,sid, ssid) to $P$, and mark record $\left(\operatorname{ssid}, P, P^{\prime}, p w\right)$ completed.

Figure 8: Ideal functionality $\mathcal{F}_{\text {apwKE }}$ for verifier-based PAKE with explicit authentication from [GMR06]. Note that we applied a single wording change (underlined) by replacing Impersonate with Svrlmpersonate for making it more explicit that this message models impersonation of the server. 


\section{The Asymmetric AuCPace protocol}

Setup: This protocol uses a random oracle functionality $\mathcal{F}_{\text {RO }}$ for all of the hash functions $\mathrm{H}_{3}, \mathrm{H}_{4}, \mathrm{H}_{5}$ and the password hash PBKDF $\sigma\left(\mathrm{H}_{6}\right)$ with a parametrization $\sigma$ and salt size of $m_{s}$ bits. The protocol also uses a balanced PAKE functionality $\mathcal{F}_{\text {pwKE }}$ as well as a Diffie-Hellman key exchange protocol (written in exponentiation notation $X^{y}$ ) operating on base point $B$ and group order $m_{\mathcal{J}}$ working on a cryptographic sub-group $\mathcal{J}$ of an elliptic curve and its quadratic twist $\mathcal{J}^{\prime}$.

\section{Password storage protocol:}

When $P_{i}$ (who is a client) is activated using StorePWfile $\left(s i d, P_{j}, p w\right)$ for the first time, he does the following. He samples a fresh value salt $\leftarrow \$\{0,1\}^{m_{s}}$, calculates the password hash $w=\mathrm{H}_{6}\left(\right.$ salt $\left.\|\sigma\| p w \| P_{i}\right)$ by using $\mathcal{F}_{\mathrm{RO}}$. He then calculates a Diffie-Hellman point $W=B^{w}$.

He sends a message (StorePWfileSvr, sid, $P_{i}$, salt, $\sigma, W$ ) to $P_{j}$. When $P_{j}$ which is a server receives (StorePWfileSvr, sid, $P_{i}$,salt, $\sigma, W$ ) from $P_{i}$ for the first time, he sets file $[$ sid $]=\left(\right.$ sid,salt, $\left.\sigma, W, P_{i}\right)$.

\section{Protocol steps for session establishment:}

1. When $P_{j}$ receives input (SvrSession, sid, ssid, $P_{i}$ ), he sets up a session record $\left(\right.$ sid, ssid, $\left.P_{i}\right)$ and marks it as fresh. He then waits for a (username, sid, ssid, $P_{i}$ ) message.

2. When $P_{i}$ receives input (CltSession, sid, ssid, $\left.P_{j}, p w\right)$ he sets up a session record $\left(\right.$ sid, ssid, $P_{j}$ ) and marks it as fresh. He then sends message (username, sid, ssid, $P_{i}$ ) to $P_{j}$ and awaits a response.

3. When $P_{j}$ receives input (username, sid, ssid, $P_{i}$ ), he obtains the tuple stored in file $[s i d]$ (aborting and marking the session record as completed if this value is not properly defined). He then samples a fresh nonzero exponent $x$ with $0<x<m_{\mathcal{J}}$ and calculates $X=B^{x} . \quad P_{j}$ then sends (hashingParams, sid, ssid, $\sigma$,salt, $X$ ) to $P_{i}$. $P_{j}$ then calculates $W^{x}$. He then sends (NewSession, (sid, ssid), $P_{j}, P_{i},\left(\right.$ sid, ssid, $\left.\left.W^{x}\right)\right)$ to $\mathcal{F}_{\text {pwKe }}$ and awaits a response.

4. When $P_{i}$ receives input (hashingParams, sid, ssid, $\sigma$,salt, $X$ ) he verifies $\mathrm{X}$ and calculates $w=\mathrm{H}_{6}\left(\right.$ salt $\left.\|\sigma\| p w \| P_{i}\right)$. He then calculates $X^{w}$. He then sends (NewSession, (sid, ssid), $P_{i}, P_{j},\left(\right.$ sid, ssid, $\left.\left.X^{w}\right)\right)$ to $\mathcal{F}_{\text {pwKe }}$ and awaits a response.

5. When $P_{j}$ receives input ((sid, ssid), sk1) he calculates $T_{a}=\mathrm{H}_{3}(s k 1), T_{b}^{\prime}=\mathrm{H}_{4}(s k 1)$ and $s k=\mathrm{H}_{5}(s k 1)$ and adds $T_{a}, T_{b}^{\prime}, s k$ to the session record. He then sends (Authenticator, sid, ssid, $T_{a}$ ) to $P_{i}$ and awaits a response.

6. When $P_{i}$ receives input ((sid, ssid), sk1) he calculates $T_{a}^{\prime}=\mathrm{H}_{3}(s k 1), T_{b}=\mathrm{H}_{4}(s k 1)$ and $s k=\mathrm{H}_{5}(s k 1)$ and adds $T_{a}^{\prime}, T_{b}, s k$ to the session record. Then he sends (Authenticator, sid, ssid, $T_{b}$ ) to $P_{j}$ and outputs (sid, ssid,sk). He then waits for a response.

7. When $P_{i}$ receives a message (Authenticator, sid, ssid, $T_{a}$ ) he compares $T_{a}^{\prime}$ with $T_{a}$ and aborts in case of differences. Else $P_{i}$ outputs $($ sid, ssid, sk).

8. When $P_{j}$ receives a message (Authenticator, sid, ssid, $T_{b}$ ) he compares $T_{b}^{\prime}$ with $T_{b}$ and aborts in case of differences. Else $P_{j}$ outputs (sid, ssid, sk).

Stealing the password file: When $P_{j}$ (who is a server) receives a message (StealPWfile, sid, $P_{j}, P_{i}$ ) from the adversary $\mathcal{A}$, if file $[s i d]$ is defined, $P_{j}$ sends it to $\mathcal{A}$.

Figure 9: AuCPace Protocol definition for the proof of indistinguishability. 
simulators $\mathcal{S}_{0}$ to $\mathcal{S}_{4}$ which implement part of their functionality in a subroutine library $\mathcal{F}_{0}$ to $\mathcal{F}_{4}$, where $\mathcal{F}_{4}$ exactly implements the ideal functionality $\mathcal{F}_{\text {apwKE. }}$. Throughout this proof, we show that all of the individual games are indistinguishable for $\mathcal{Z}$.

\subsection{Game-based proof}

Theorem 2. The protocol from figure 9 securely realizes $\mathcal{F}_{\text {apwKE }}$ in the $\left(\mathcal{F}_{R O}, \mathcal{F}_{\text {pwKE }}\right)$ hybrid model in the presence of adaptive adversaries under the assumption of the hardness of the computational Diffie-Hellman problem in $\mathcal{J}$ (A3).

\subsubsection{Game $\mathrm{G}_{0}$ : Real Game}

$\mathrm{G}_{0}$ is the real game in the random-oracle model using the functionality $\mathcal{F}_{\mathrm{RO}}$ from figure 7 for calculating the password hash PBKDF. Honest parties $P_{i}$ execute the actions of the real-world protocol until eventually getting corrupted. Specifically client entities $P_{i}$ receive StorePWfile and $\mathrm{CltSession}$ queries from the environment $\mathcal{Z}$ and return session keys upon success. Server entities $P_{j}$ receive SvrSession queries. On the event of corruptions, all the internal state of the parties is passed to the real-world adversary algorithm $\mathcal{A}$, specifically for server corruptions, the password verifier $W$ is returned. The subroutine library $\mathcal{F}_{0}$ is empty.

\subsubsection{Game $G_{1}$ : Modeling the random oracle for the hash}

In $G_{1}$ we replace calls to $\mathcal{F}_{\mathrm{RO}}$ by a separate implementation of the random oracle for PBKDF and the hash functions in a straight-forward way. Again we maintain an initially empty list $\Lambda$ of value pairs $(n, q, r)$. For any hash query $\mathrm{H}_{n}(q)$ such that $(n, q, r)$ appears in $\Lambda$ from any of the ITI, the returned answer is $r$. In case that no query $q$ has yet occurred we implement the conventional random-oracle model by choosing a new random $r$ of length $k$, by storing $(n, q, 0, r)$ in $\Lambda$ and by returning $r$ to the calling ITI.

This game is indistinguishable from game $\mathrm{G}_{0}$ since the hash results both come from the same uniform distribution.

\subsubsection{Game $\mathrm{G}_{2}$ : Getting rid of the case where the adversary $\mathcal{A}$ wins by chance.}

This game is almost as the previous, only we allow the simulator to abort in case that the adversary manages to guess one of the authenticator messages $T_{a}$ or $T_{b}$ or the final session key $s k$ without querying the random oracles for $s k 1$. This happens with negligible probability, so Game $\mathrm{G}_{1}$ and $\mathrm{G}_{2}$ are indistinguishable for $\mathcal{Z}$.

\subsubsection{Game $\mathrm{G}_{3}$ : Handle mutual authentication.}

In this game we deal with mutual authentication but still allow the simulator access to the clear-text password $p w$ upon server compromise events, i.e., in this game, we do not give the simulator access to the password but instead pass the password from a StorePWfile and CltSession query to code within $\mathcal{F}_{3}$ with an implementation according to $\mathcal{F}_{\text {apwKE }}$. Temporarily, we allow $\mathcal{F}_{3}$ to return the clear-text password upon the StealPWfile query.

This way, the simulator may no longer access the password for the message StorePWfile sent from the client to the server. Since we assume that neither impersonation nor eavesdropping or message modification is feasible for $\mathcal{A}$ in this sub-step, simulation of the message provides no difficulties. We just sample a new random salt value and let the client send a message (StorePWfile, sid,salt, $\sigma, P_{j}$ ) with only the hashing parameter but without password verifier $W$. Since $\mathcal{A}$ is not allowed to eavesdrop this is indistinguishable from game $\mathrm{G}_{2}$ for $\mathcal{Z}$. 
Simulation of the (username, sid, ssid, $P_{i}$ ), does only include publicly known information and is simulated as in the real world protocol. The same holds for the server's reply (hashingParams, sid, ssid, $\sigma$, salt, $X$ ) we sample a fresh random secret scalar $x$ and salt value and calculate the public key in the message as $X=B^{x}$. Point verification for $X$ may be implemented just as in $\mathrm{G}_{2}$.

In case of compromising the server's password file, we have to return password verifiers $W$ in order to maintain indistinguishability with game $\mathrm{G}_{2}$. In game $\mathrm{G}_{3}$ we do so, by retrieving the clear-text password $p w$ from $\mathcal{F}_{3}$ and by calculating the password verifier as in the original protocol.

For simulating the authenticator messages $T_{a}$ and $T_{b}$ we sample two random values and transmit these. Since also in game $G_{2}$ these values came from a uniform distribution, the authenticator messages from game $G_{3}$ are indistinguishable from $G_{2}$ for $\mathcal{Z}$.

After sending the authenticator messages, we call the TestAbort queries of $\mathcal{F}_{3}$ for both parties and call a NewKey query upon success. In case that the adversary did destructively modify the hashingParams or the authenticator messages, we let protocol parties abort.

Games $G_{2}$ and $G_{3}$ are indistinguishable for the environment. In both games, the client aborts, if the group order of the point $X$ is small. The Diffie-Hellman points $W^{x}$ and $X^{w}$ match, thus, iff $W$ has been calculated from $B^{w}$ and $X$ has been calculated from $B^{x}$. Therefore any modification of $X$ by $\mathcal{A}$ leads to different $P R S$ strings, i.e., the input to $\mathcal{F}_{\text {pwKe }}$ matches iff the passwords used for the StorePWfile request for the client match the one from the CltSession request. As a consequence the session keys returned by $\mathcal{F}_{\text {pwKE }}$ match only if the very same passwords match. Verification of the authenticators in $\mathrm{G}_{2}$ succeeds iff $\mathcal{F}_{\text {pwKE }}$ returned the same session key to both parties. Upon any modification of the authenticator messages by $\mathcal{A}$ the parties abort in both games.

\subsubsection{Game $\mathrm{G}_{4}$ : Keeping the password secret}

In this game we disallow the simulator to access the clear-text password upon server compromise events. In this game, we add a "re-program-offline-query" list $\Lambda_{1}$ to the implementation of the random oracle in addition to its list $\Lambda$. We change the implementation as follows. Upon a query $q$ to the hash oracle, if no corresponding entry is found in $\Lambda$, we first parse salt value $P_{i}, p w$ and $\sigma$ from the query q's encoding. For all entries $\left(\right.$ sid, $\left.P_{j}, w\right)$ contained in $\Lambda_{1}$ we execute a OfflineTestPwd query on $\mathcal{F}_{4}$ for all $P_{j}$ in case of a "correct guess" result, we program the record in $\Lambda$ for the query to the value $w$ from $\Lambda_{1}$, remove the entry $\left(\right.$ sid, $\left.P_{j}, w\right)$ from $\Lambda_{1}$ and return $w$. If after parsing the full list $\Lambda_{1}$ no "correct guess" result is returned, we sample a fresh random value $r^{\prime}$, program it to $\Lambda$ and return $r^{\prime}$.

Upon server compromise, we proceed as follows. We first make a StealPWfile query to $\mathcal{F}_{4}$. Subsequently, we iterate through the PBKDF's random oracle list entries in $\Lambda$, parse the stored queries for the client id, salt, $\sigma$ and the password and execute OfflineTestPwd queries to $\mathcal{F}_{4}$. In case of a "correct guess" reply, we learned the password $p w$ and can, thus, calculate the password verifier $W$ and send it to the adversary $\mathcal{A}$. Otherwise, the password hash oracle has not yet been queried. In this case, we sample a new random hash result $w$, setup a new re-program-offline-query entry $\left(\right.$ sid, $\left.P_{j}, w\right)$ for the hash oracle $\Lambda_{1}$. In this case we calculate the password verifier as $W=B^{w}$ and send it to the adversary.

This procedure allows us to later on arrange for matching password verifiers $W$ and password hashes $w$.

We also have to handle the case of impersonation. If $\mathcal{A}$ uses the stolen password verifier in his attack strategy for impersonating a server, we let the simulator make calls to Svrlmpersonate.

The only difference to game $\mathrm{G}_{3}$ shows up with respect to the way that the password verifier $W$ is calculated. Irrespectively, whether the adversary had queried the hash oracle before the server compromise operation or after, the simulator always returns $(w, W)$ pairs matching to the respective passwords. Also in both games the distribution of password 
hashes and verifiers $w$ and $W$ is the same. Game $\mathrm{G}_{3}$ and $\mathrm{G}_{4}$ are, thus, indistinguishable for the environment $\mathcal{Z}$.

The real-world protocol AuCPace, thus emulates the ideal functionality $\mathcal{F}_{\text {apwKE }}$ in the $\mathcal{F}_{\text {pwKE }}, \mathcal{F}_{\text {RO }}$ hybrid model.

\section{Partial augmentation}

\subsection{The ideal functionality $\mathcal{F}_{\text {papwKE }}$ for modeling partial augmentation.}

For the proof we introduce a new concept for partial augmentation of a PAKE protocol. The corresponding functionality is depicted in figure 10 . In comparison to $\mathcal{F}_{\text {apwKe }}$ partial augmentation $\left(\mathcal{F}_{\text {papwKE }}\right)$ gives the attacker the possibility to also impersonate the client after having succeeded in compromising the server.

In the partially augmented variant of our protocol, we replace the server-chosen ephemeral key-pair $(x, X)$ by a long-term key pair that is re-used over several login sessions (same sid, different ssid). We would have liked to choose the key-pair only once at the point, where the server's Turing machine is first instantiated and not upon each password configuration. Unfortunately this is technically not possible in the UC framework, since this would correspond to a shared state over several sid. For this reason, we need to let the server choose $(x, X)$ upon password configuration and store $W^{x}$ together with $X$ in the password file, i.e., just as the password verifier, the public key $X$ becomes part of the shared state for session sid. Note that using a long-term public key essentially halves the computational complexity of AuCPace for the server for the case of the login sessions.

At a first glance, since after a server $P_{j}$ 's compromise, none of the security guarantees with respect to the adversary are maintained, it might be argued that $\mathcal{F}_{\text {papwKe }}$ does not actually provide any meaningful advantage in comparison to $\mathcal{F}_{\text {pwKE }}$.

The advantage, however, becomes obvious when considering the IIoT setting with more than two servers sharing the same user credentials. In fact after executing a StealPWfile query on server $P_{j}$ the adversary has full control over $P_{j}$. Note, however that the adversary is not given the clear-text password $p w$ from $P_{j}$ upon server compromise. He is only granted the capability to execute OfflineTestPwd queries.

In settings where the adversary may expect other server entities $P_{k}$ to operate with the same password $p w$ as $P_{j}$, client impersonation for connections with $P_{k}$ is still precluded.

Note that this is occurring exactly in the use-case of industrial control plants. There user credentials (password verifiers) may be shared by many small server entities, which may be comparably easily stolen/compromised. In this setting, server compromise might most likely be implemented by invasive attacks on the hardware, e.g., by stealing a first server, un-soldering microcontroller or memory chips and by side-channel attacks that re-open debug ports. In this setting $\mathcal{F}_{\text {papwKe }}$ provides very meaningful protection to the honest subset of servers. It might be likely to detect theft of the device and the partial augmentation feature might provide a sufficiently large time-window allowing for changing user credentials on the plant.

Also undetected re-insertion of a compromised server in a plant may not be a relevant attack scenario, such that the additional capability of the adversary to impersonate the client on this specific server does not actually degrade the security in practice. Moreover, as we will show, the AuCPace scheme allows for a server-specific configuration for partial and full augmentation. A server entity where non-invasive attacks allowing for a re-insertion into an installation should be considered feasible might choose to implement $\mathcal{F}_{\text {apwKE }}$ using AuCPace with ephemeral key pair $(x, X)$ while a server where a more invasive attack is presumed necessary in order to compromise the database (leading to device destruction) might choose to use a long-term secret $x$ and as a consequence $\mathcal{F}_{\text {papwKe }}$.

Similar security guarantees of $\mathcal{F}_{\text {papwke }}$ could also be realized if any server uses a 
The functionality $\mathcal{F}_{\text {papwKe }}$ is an extension to the functionality $\mathcal{F}_{\text {apwKe }}$ from figure 8. It implements all of the $\mathcal{F}_{\text {apwKE }}$ queries and extends the capabilities of the adversaries by the following query:

Upon receiving a query (Clt Impersonate, sid, ssid) from adversary $\mathcal{S}$ :

If there is a session record of the form $\left(s s i d, P_{i}, P_{j}, p w\right)$ which is fresh, then do: If there is a password data record (file, $P_{i}, P_{j}, p w$ ) that is marked compromised, mark the session record compromised and reply to $\mathcal{S}$ with "correct guess", else mark the the session record interrupted and reply with "wrong guess".

Figure 10: Ideal functionality $\mathcal{F}_{\text {papwKE }}$ for partial verifier-based PAKE with explicit authentication.

different "salt" value for each client, e.g., by letting the server provide a random salt value upon password configuration. This, however precludes mechanisms offering an off-line user credential distribution because all server identities need to be known at the time when the user configures his password. It would not allow for the flexibility to asynchronously add further servers to a plant after password registration. Note also that this way upon password changes, the complex $\mathrm{PBKDF}_{\sigma}$ password hash would have to be calculated once for each server, significantly reducing the feasible strength of the workload parameters $\sigma$.

For the same reason "personalizing" a password hash for a server by hashing it together with the server ID provides weaker security guarantees than partially augmented AuCPace. Either the capability to add new servers to a plant after password registration is lost or a central password distribution server would be required to hold information allowing for impersonating any user. In the AuCPace context the distribution server would only hold information on $W$ not allowing for impersonation attacks without offline dictionary attacks because entering the protocol in client role requires the password-derived scalar $w$.

\subsection{Proof}

Theorem 3. The protocol from figure 9 with using a long-term key-pair $(x, X)$ instead of the ephemeral key pair from step 3 in figure 9 securely realizes $\mathcal{F}_{\text {papwKE }}$ in the $\left(\mathcal{F}_{R O}, \mathcal{F}_{\text {pwKE }}\right)$ hybrid model in the presence of adaptive adversaries under the assumption of the hardness of the computational Diffie-Hellman problem in $\mathcal{J}$ and $\mathcal{J}^{\prime}$.

We implement the proof for the partially augmented protocol in the UC hybrid model, just as for the fully augmented variant. However, here leave the black-box model for $\mathcal{A}$ for simplicity.

Since we assume that the key pair $(x, X)$ is used for several protocol runs, we give the adversary access to the secret exponent $x$ upon server compromise. For this reason, we also have to consider adversaries $\mathcal{A}$ which base their attack strategy on this knowledge. In this case, the adversary is able to calculate the password related string $P R S$ and, thus, impersonate the client. In case of such an attack strategy, we let the simulator use the CltImpersonate query of $\mathcal{F}_{\text {papwKE }}$ in order to make the ideal and real world indistinguishable for the environment $\mathcal{Z}$.

\section{Performance assessment of the AuCPace protocol}

In this section, we will discuss the properties of AuCPace and other V-PAKE constructions from the perspective of extremely resource-constrained servers for industrial installations.

Fairly benchmarking algorithms and protocols often is a difficult task since many aspects need to be considered. Advantages regarding one side could often be obtained only at the cost of disadvantages elsewhere. 
Since a large number of PAKE protocols have previously been suggested (see, e.g., [SOAA15, PW17] for a larger overview), we saw the need to focus here on a reduced subset of constructions considered suitable according to clear criteria. Our findings are summarized in table 1. For this discussion we concentrated on protocols fulfilling the following constraints.

- We restricted our analysis to constructions coming with explicit security proofs.

- We concentrated on constructions requiring "few" exponentiations because in our setting everything but the most efficient constructions were considered impractical. Essentially this requirement is equivalent to only considering constructions proven to be secure in the random oracle model. For the very same reason, we did not consider protocols that mandatorily require comparably costly large-characteristic field operations or pairings (or were only proven secure in this setting).

- We only considered V-PAKE schemes because we believe server compromise to be a highly relevant attack, specifically for plants with large outdoor installations. In this case in our opinion the additional mitigations provided by V-PAKE could prove beneficial, particularly when considered in conjunction with strong password hashes and password policies enforcing minimum entropy levels.

- We also considered proven forward secrecy to be mandatory for future-proof concepts since in important settings the secure channel established by the V-PAKE protocol will be used for securely changing passwords.

Specifically the requirement of proven forward secrecy turned out to be quite restrictive. Unfortunately many otherwise very interesting constructions, such as notably AugPake [SKI10] and SPAKE2+ [CKS08] do not offer this feature [PW17].

\subsection{Discussion of the comparison overview}

In table 1 we summarize our results. For a fair comparison, the message count does not include the initial messages for session-id generation that we believe mandatory for all of the UC-secure protocols and only consider variants providing explicit mutual authentication.

Message counts for password registration consider the recommended procedure from the respective authors. The computational complexity comparison distinguishes fixed (f) and variable-point (v) scalar multiplications as well as group order field inversions (i).

The size estimate of password verifiers to store in the server's database assumes 32 bytes (B) for each of username, associated data for granted user authorizations and salt and compressed elliptic curve points for the 128 bit security level. We estimated 32 byte storage overhead for the overhead for symmetric authenticated encryption.

\subsection{Security guarantees}

While AuCPace and OPAQUE were both analyzed within the UC framework, the security proof for VTBPEKE relies on the BPR model and derives somewhat more quantitative upper bounds for the possible adversary advantage.

To some extend, the security guarantees depend on the framework used for the security analysis. For instance, unlike for the BPR-model, in the UC model used here and in [GMR06, JKX18] no assumptions regarding password distributions apply.

One minor difference might be that for AuCPace, we did consider fully adaptive adversaries during session establishment, while the other UC-based approaches [GMR06, JKX18] only considered static server corruptions.

However, according to our assessment in the view of practical relevance for real-world applications, we believe that these differences among the filtered candidates within table 
Table 1: Comparison of different V-PAKE constructions.

\begin{tabular}{r|cccc} 
& $\begin{array}{c}\text { AuCPace } \\
\text { (part. })\end{array}$ & AuCPace & VTBPEKE & OPAQUE \\
\hline message count & 4 & 4 & 3 & 3 \\
message count pw-Registr. & $1 \mathrm{c}$ & $1 \mathrm{c}$ & $1 \mathrm{c}$ & $1 \mathrm{~s}+2 \mathrm{c}$ \\
\hline precomp. res. & no & no & no & yes \\
proof & $\mathrm{UC}$ & $\mathrm{UC}$ & $\mathrm{BPR}(\mathrm{ROR})$ & $\mathrm{UC}$ \\
\hline comp. complexity server & $2 \mathrm{v}$ & $3 \mathrm{v}+1 \mathrm{f}$ & $3 \mathrm{v}+1 \mathrm{f}+1 \mathrm{i}$ & $3 \mathrm{v}+1 \mathrm{f}$ \\
comp. complexity client & $3 \mathrm{v}$ & $3 \mathrm{v}$ & $3 \mathrm{v}+1 \mathrm{f}$ & $4 \mathrm{v}+1 \mathrm{f}$ \\
\hline$x$-coordinate only & possible & possible & - & - \\
simplified point ver. & possible & possible & - & - \\
\hline pw-verifier size estimate & $\approx 96 \mathrm{~B}$ & $\approx 64 \mathrm{~B}$ & $\approx 64 \mathrm{~B}$ & $\approx 280 \mathrm{~B}$ \\
total message size estimate & $\approx 160 \mathrm{~B}$ & $\approx 160 \mathrm{~B}$ & $\approx 160 \mathrm{~B}$ & $\approx 280 \mathrm{~B}$ \\
\hline Map2Point necessary & yes & yes & no & yes \\
\hline
\end{tabular}

1 might be of somewhat minor importance. Among these four constructions, we only identified two significant security guarantee differences.

Firstly, the unique feature of OPAQUE is that it allows for starting with the offline attack only after compromising the server (pre-computation resistance). With respect to this feature OPAQUE, provides stronger guarantees. However this, as will be derived below, comes at the cost of reduced flexibility in the password registration phase.

Secondly, the unique feature of AuCPace is that it optionally allows for partial augmentation allowing for significantly less computational complexity than the other candidate protocols. This comes at the cost that in case of a corrupted server, the security guarantees correspond only to those of a balanced PAKE, while the V-PAKE guarantees are only maintained for other, yet uncompromised servers, sharing the same password database.

\subsection{Computational efficiency for constrained servers}

When considering the fully augmented setting all of the protocols in table 1 require four exponentiations per session for the constrained server. In case of OPAQUE and the fully augmented AuCPace one of these is a fixed-base point operation and could be pre-computed. The perceived delay on the HMI interface due to the complex scalar multiplications will correspond, thus, to only three scalar multiplications in the case of AuCPace and OPAQUE in contrast to four in the case of VTBPEKE.

It is worth noting, that an important part of the efficiency of OPAQUE could be attributed to the use of the highly efficient HMQV [Kra05] construction. Unfortunately HMQV is covered by patents in some important countries. When replacing HMQV with an alternative AKE not covered by patents, such as, e.g., NAXOS [LLM07], a corresponding OPAQUE-like construction would likely require five exponentiations. The problem of HMQV patents has been identified and possibly in the future HMQV might be freely usable [Kra18] for OPAQUE at least for some applications but, unfortunately, this is presently still uncertain.

We believe that many applications might be scared away from algorithms using patented components, not only because of the cost but also when considering complex licensing agreements and reporting duties.

For important applications in the IIoT setting, we conjecture that adequate security could be obtained also when implementing partial augmentation. Specifically here we 
consider settings where server compromise involves stealing of hardware and highly invasive physical attacks likely to destroy hardware.

In the partially augmented setting, AuCPace has a computational complexity of two exponentiations and provides the most efficient solution among all known verifier-based PAKE protocols. Attacks on yet un-compromised servers are prevented even if these are working with the same password verifier $W$ as, e.g., distributed by a centralized database server.

\subsection{Implementation effort}

Both, OPAQUE and VTBPEKE require, in contrast to AuCPace, a full group structure for actual implementation, i.e., both, $x$ and $y$ coordinates of points are necessary and implementations have to deal with the point compression and point verification issues (and possibly patent trouble) if they aim at reducing the message and/or password verifier length.

AuCPace, in contrast could also be implemented by using $x$-coordinate-only DiffieHellman algorithms such as X25519. This could be used for saving both, code-ROM and RAM memories and could facilitate secure (e.g., efficient constant-time) implementation.

While beside the elliptic-curve operations AuCPace only requires a cryptographic hash, the other protocols also all require a symmetric encryption primitive. AuCPace is, thus, somewhat simpler to implement. This, however, might be only a rather minor advantage in many settings, since most application based on PAKE protocols might anyway require symmetric authenticated encryption after successful generation of the session key.

VTBPEKE and constructions based on [GMR06] have the advantage that they could be implemented without requiring hashing to elliptic curve points. Note that in the light of intellectual property rights, this could be an advantage, specifically regarding standardized curves in short-Weierstrass form. For instance for the application in travel documents the PACE protocol [BFK09] often is implemented in it's computationally complex "generic mapping" variant. The reason for this approach, in our opinion, is the fact that this avoids any need of hashing to curve groups and thus the mere risk of patent conflicts. Note that regarding patents it is, in our opinion, not the main question whether a method is actually rightfully covered by a patent or not. In fact already uncertainty and the merely potential risk of conflicts (and possibly large costs), might often motivate users to select circumvention techniques.

For VTBPEKE, unlike AuCPace, the server also needs to implement inversions with respect to the group order, i.e., in addition to the field arithmetic a second set of modulo reductions needs to be implemented.

AuCPace, thus, allows in comparison to the other candidates for significantly improved ease-of-implementation. This possibly might reduce the risk of implementation errors.

\subsection{Bandwidth and latency aspects}

At a first glance, AuCPace requires a comparably large number of communication rounds. When considering also the initial establishment of the (sub-) session id (ssid) by exchange of the messages $t$ and $s$, we come up with a total message count of 5 . Without counting the ssid establishment, the message count amounts to 4 messages only.

Both OPAQUE and VTBPEKE require 3 messages. However in the case of OPAQUE this does not account for messages required for session id establishment. It is worth noting that according to our best knowledge and in line with the conclusions from the analysis of Fischlin, Bender and Kügler [BFK09], the security model of the UC framework assumes mandatorily that the ssid is fixed prior to initiating the protocol. Specifically, this was identified as one obstacle preventing security analysis of PACE in an UC context. The requirement of session id establishment should in fact also apply for other UC-secure 
constructions such as OPAQUE and protocols based on [GMR06]. However unlike here the corresponding message count is not considered in the respective papers. Note that for AuCPace, actually the first usage of the ssid occurs only when entering the CPace protocol steps.

We believe that the typical use-case of a PAKE protocol is establishment of a secure (encrypted and authenticated) channel using the session key. In this case, the final two authenticator messages are optional, just as for OPAQUE, reducing the message count to 3 and 2 respectively for AuCPace and OPAQUE when disregarding session id establishment. Note that for both, OPAQUE and AuCPace the two explicit authentication messages are not mandatory for UC-securely implementing the $\mathcal{F}_{\text {apwKE }}$ functionality.

In comparison to AuCPace, OPAQUE and VTBPEKE require one and two messages less, respectively, when considering session id generation. As a result, message latency will add up a bit more for AuCPace. However, in comparison to OPAQUE and [GMR06], messages used for AuCPace and VTBPEKE are significantly shorter. Specifically, it is not necessary to transfer encrypted (and authenticated) versions of public-private key pairs. Note that this provides an advantage when using the PAKE protocol over a low-bandwidth wireless link, specifically if very small packets are used on the physical layer, such as in case, e.g., for the bluetooth-low-energy standard. Note also, that the shorter messages allow for reduced buffer sizes and all-over reduced RAM memory requirements.

AuCPace allows for pipelining message transfer and cryptographic calculation, improving upon user-experienced latency. For instance, the server may interleave transmission of the last message from the augmentation layer $(X, \sigma$, salt $)$ and calculation of the public point $Y_{a}$, such that $Y_{a}$ is transmitted later in a separate message. In settings where message delivery latency is significant and computation is fast, however, the server may choose to include $Y_{a}$ in the earlier message.

\subsection{Intellectual property rights}

We believe that pending patents on algorithms and algorithmic substeps might seriously hamper actual use of a cryptographic protocol. AuCPace was specifically designed for avoiding all patents known to the authors. We believe that this also applies to VTBPEKE. The only aspect where we are aware of the potential of conflicts for AuCPace is the Map2Point substep where efficient algorithms might possibly have to be analyzed in detail, specifically for curves in short Weierstrass form. For this reason, we will sketch a circumvention approach in the appendix.

\subsection{Registering verifiers}

Regarding password verifier registration on the server we identify two main aspects: Size of the password verifiers on the server and flexibility regarding password-verifier registration protocols.

$\mathrm{AuCPace}$ is characterized by requiring only very little persistent storage for the password verifiers, i.e., in addition to the user identifiers, the encoding of the user's authorization and the salt value (which might require roughly 32 bytes), only one (two) group elements requiring typically 32 bytes need to be stored for full (partial) augmentation. In total an amount of 64 (96) bytes suffices. (Possibly further future analysis, e.g. in the BPR model could reduce the verifier size for partially augmented variants also to 64 bytes.)

Other protocols, specifically [GMR06, JKX18] require significantly longer verifiers. For instance, OPAQUE requires seven group elements or secret scalars, three of which are included in an encrypted authenticated structure (typically requiring additional nonce and MAC fields with, e.g,. 2x16 bytes). Even when considering point compression, this could easily add up to a total verifier size of $>280$ bytes. 
Note that the size of password verifiers should not be disregarded. Some microcontrollers include small amounts (e.g., 1 kByte) of somewhat protected memory (e.g., tamperprotected RAM) meant to be used for storing sensitive information such as cryptographic keys or password-related information. Excessive size of password verifiers might require additional complexity in the application code or make implementers be tempted to use unprotected memory also on devices that might be exposed to physical attacks. Note also that write operations on persistent memory could generate large power consumption transients.

In our opinion, the fact that AuCPace only needs fairly short password verifiers is strongly linked to the fact that it is a sequential scheme executing Diffie-Hellman before the SPEKE-like substep and not in parallel. In schemes using a large amount of parallel operations, such as for instance SPAKE2+ [CKS08] or OPAQUE [JKX18] much more complex password verifiers have to be used.

Regarding OPAQUE, it is worth to draw attention to a side-effect of the security property of pre-computation attack resistance. This desirable feature is realized by executing the complex password hash after finishing the protocol step of the oblivious pseudo-random function (OPRF). This forces the server as a consequence to maintain an online connection to the client upon password changes if the server is incapable of calculating the password hash. First an interaction is required between client and server for calculating the OPRF which is required as pre-requisite for calculating the computationally complex PBKDF password hash. In other words, for OPAQUE password registration requires bi-directional communication. Otherwise we would have to let the client entity choose the secret server scalar for the OPRF upon password configuration, which we do not consider ideal from a security perspective (in line with the suggestion of the authors of [JKX18]).

As a consequence, we believe that for OPAQUE, user credential distribution protocols based on offline tickets (allowing for only one unidirectional message) might be much more difficult to implement. Password registration for AuCPace could, in contrast be implemented more flexibly by one single unidirectional message. This comes, however at the cost that pre-computation attacks could not be prevented.

\section{Implementation on ARM Cortex M0 and M4 microcon- trollers}

One important target platform for resource-constrained (I)IoT devices are 32 bit microcontrollers, such as the ARM Cortex M0 and Cortex M4 series. We have implemented AuCPace25519 in its partially augmented variant on nRF51 and nRF52 microcontrollers from the company Nordic Semiconductors and three different microcontrollers from the manufacturer ST Microelectronics.

In this section we first describe the high-level strategy for implementing the X25519 Diffie-Hellman protocol and Elligator2. The method that we employ for Elligator2 has already been presented in $\left[\mathrm{BDL}^{+} 11\right.$, BHKL13], but we considered it helpful to write it down explicitly here, because we were aware of it only after having received a corresponding hint [Ham17]. Subsequently, we will elaborate on our strategy for implementing the field arithmetic.

\subsection{Implementation of $\times 25519$}

AuCPace25519 uses X25519 for generating the password verifier and the session key $s k 1$. We make use of the constant-time Montgomery ladder algorithm from $\left[\mathrm{DHH}^{+} 15\right]$. Both fixed-point and variable-point scalar multiplication require 1287 field multiplications and 1274 field squarings. We did implement two variants. Firstly for the sake of comparison with 
related work, we implemented a synchronous version of the X25519 function. Secondly, we implemented a second, asynchronous version of X25519. For the latter we implemented an asynchronous cryptographic engine (ACE) object that stores the intermediate state of the scalar multiplication. This allows our implementation to suspend and resume calculations after each ladder step in case that the power budget requires the microcontroller to enter a sleep mode.

We came to the conclusion, in line with findings from [Ham12], that the constant-time Montgomery ladder is, most probably the most efficient known algorithmic choice available for Diffie-Hellman on Curve25519, if we aim at avoiding memory consuming pre-computed tables.

\subsection{Implementation of Elligator2}

In order to remain consistent with the notation used in [BHKL13] we denote the Legendre symbol that records quadratic residuosity of $a \bmod q$ (with $q$ being an odd prime number) with $\chi$ :

$$
\chi(a) \triangleq\left(\frac{a}{q}\right) \equiv a^{\frac{q-1}{2}}
$$

Remember that the Elligator2's decoding function for a Weierstrass curve $E: y^{2}=$ $x^{3}+A x^{2}+B x$ is the function $\psi: R \rightarrow E\left(\mathbb{F}_{q}\right)$ defined as follows: $\psi(0)=(0,0)$; if $r \neq 0$ then $\psi(r)=(x, y)$ (see [BHKL13]). For a set $R$ defined as

$$
R \triangleq\left\{r \in \mathbb{F}_{q}: 1+u r^{2} \neq 0, A^{2} u r^{2} \neq B\left(1+u r^{2}\right)^{2}\right\}
$$

the following elements of $\mathbb{F}_{q}$ are defined (we use only $x$ coordinates):

$$
\begin{aligned}
v & =\frac{-A}{1+u r^{2}} \\
\varepsilon & =\chi\left(v^{3}+A v^{2}+B v\right) \\
x & =\varepsilon v-(1-\epsilon) \frac{A}{2}
\end{aligned}
$$

In case of Curve25519, $q=2^{255}-19, A=486662$ and $B=1$. Also $u$ is chosen to be 2 . If we would calculate $x$ directly, we would need two exponentiations, one for the inversion (3) and one for the Legendre symbol $\chi(4)$. In fact computing a single exponentiation is enough, when using the inverse square root algorithm from [BDL $\left.{ }^{+} 11, \mathrm{BHKL} 13\right]$. When substituting $v$ in $v^{3}+A v^{2}+B v$ one obtains, in a projective representation, the fraction

$$
\frac{a}{b} \triangleq \frac{A^{3} u r^{2}+A B\left(1+u r^{2}\right)^{2}}{\left(1+u r^{2}\right)^{3}}
$$

As a property of the Legendre symbol we have:

$$
\chi\left(\frac{a}{b}\right)=\chi(a b)
$$

since $\chi\left(\frac{a}{b}\right) \equiv a^{\frac{q-1}{2}} b^{\frac{-(q-1)}{2}} \equiv a^{\frac{q-1}{2}} b^{\frac{q-1}{2}} \equiv \chi(a b)$ with $1 \equiv a^{q-1}(\bmod q)$ (Fermat's little theorem). Now let's define

$$
\begin{aligned}
& c \triangleq a b \\
& d \triangleq 1+u r^{2} \\
& s \triangleq\left(c d^{2}\right)^{\frac{q-3}{2}}
\end{aligned}
$$


We have $s^{2} c d^{2}=\left(c d^{2}\right)^{q-2}=\frac{1}{c d^{2}}$ using Fermat's little theorem again. So the inverse is given by:

$$
\frac{1}{d}=s^{2} c d^{2} c d
$$

iff $c \neq 0$ and $d \neq 0$. If $c=0$, then the point is $(0,0)$ or $\infty$. If $d=0$ the point is $\infty$ and we return 0 in either case. On the other hand it could be written $s c d^{2}=\left(c d^{2}\right)^{\frac{q-1}{2}} \equiv \chi\left(c d^{2}\right)$. Furthermore it holds that by definition of the Legendre symbol:

$$
\chi(a b)=\chi(a) \chi(b)
$$

and

$$
\chi\left(d^{2}\right)=1
$$

unless $d=0$. So

$$
s c d^{2} \equiv \chi\left(c d^{2}\right)=\chi(c)=\chi(a b)=\chi\left(\frac{a}{b}\right)
$$

By placing (14) into (11) we get

$$
\frac{1}{d}=\chi\left(\frac{a}{b}\right) s c d
$$

This means that the Legendre symbol and the inverse (and finally Elligator2) was calculated by means of a single exponentiation (10). In case of Elligator2 for Curve25519 the algorithm requires a total of 254 field squarings and 23 field multiplications.

\subsection{Implementation of the field arithmetic}

The implementations for Cortex M0 and M4 share all of the group arithmetic and highlevel algorithms but rely on separate field arithmetic for addition, subtraction, negation, multiplication and squaring.

Our implementation for the Cortex M0 uses the same highly optimized field arithmetic as in $\left[\mathrm{DHH}^{+} 15, \mathrm{HL} 17\right]$. Here register pressure and the limited capability of the multiplier engine providing only 32 bits of a result make it beneficial to employ three cascaded Karatsuba stages. The implementation for the Cortex M4 makes use of a new, yet unpublished optimized implementation. Just as for the M0, we use a packed radix 32 representation. Throughout the implementation we reduce field elements modulo $2^{256}-38$.

Our implementation on the M4 uses the much more powerful UMLAL and UMAAL instructions allowing for simultaneously multiplying two 32 bit words and accumulating up to two 32 bit words. Due to the significant advantage of using several stages of Karatsuba multiplication for the M0, we have implemented several variants of Karatsuba multiplication also for the Cortex M4. Experiments, however, have shown that here the reduced register pressure (the "upper" registers R8 ... R12 and R14 may be used without restrictions) in addition to the fact that accumulation comes essentially for free made schoolbook multiplication faster when register-allocation is carefully tuned. Based on our experiments, we presume that for carefully optimized code on the M4, Karatsuba techniques might become beneficial again for integer sizes above 512 bits.

In order to optimize the register allocation, we have generated the assembly sources by a code generator handling register allocation and spill register storage on the stack. For the accumulation of intermediate results during the multiplication we also make use of the UMLAL and UMAAL instructions. Note that when one register with the value 1 is available, UMAAL allows for implementing three 32 bit additions in one single cycle 
$(r:=a+b+1 * c)$ yielding a 64 bit result. For subtraction (and for squaring), we made use of a specific architectural property of the M4 architecture. There two distinct ways of handling addition carries are possible. In addition to the UMAAL -based method above, flag-based add and add-with-carry (ADDS, ADCS) and subtract-with-borrow (SUBS, SBCS) instructions are available. The multiplication instructions are specified not to modify the addition/subtraction carry flag. We did use this for merging reduction with subtraction of field elements. We first accumulate both the value to subtract and a multiple of the prime (that stems from reduction) by using multiply-accumulate instructions. Then we use subtract with borrow to simultaneously subtract both results. Note that this will result in remarkable speed differences between addition and subtraction. Addition could be implemented very efficiently by using the powerful multiplication engine. Subtraction is somewhat slower because SUBS and SBCS have to be used.

For addition and subtraction, we did use the powerful inline assembly capabilities of both, GCC and CLANG, that allowed us to avoid a significant amount of call overhead. In order to avoid operand fetches and stores wherever possible, we also made use of an inline assembly function that merges addition of a first operand with the curve-constant's multiple of a second operand $(r:=a+b * 121666)$. Addition and subtraction of field elements follows the strategy from $\left[\mathrm{DHH}^{+} 15\right]$ by first processing the most significant word and then merging reduction of the two most significant bits and addition(subtraction) operation for the remaining seven words. We make use of the available carry bit 255 to obtain an implementation with only one single carry chain.

One additional optimization strategy was to bundle load and store operations together as much as possible in blocks. This way the pipeline latency on the M4 could be reduced. Isolated load and store operations account for two clock cycles each, while a sequence of $n$ such operations only accounts for $n+1$ cycles.

\subsubsection{Field multiplication for ARM Cortex M4}

Table 2 depicts the sequence $(0 \ldots 63)$ in which each of the 64 partial products of the schoolbook multiplication of the input operand words A0 .. A7 and B0 .. B7 is executed. Our optimization of the multiplication strategy does not seem to follow a regular pattern at first sight.

We use this sequence several reasons. Firstly, we observed that keeping as many input operands in registers as possible is equally important as avoiding stack spills of intermediate multiplication results. Secondly, it is worth noting that a multiplication actually costs fewer instructions if two intermediate results are to be accumulated at the same time. If only one intermediate result is to be accumulated, a MULAL instruction has to be used, which typically requires clearing of a scratch register ( +1 instruction).

Basically four subblocks may be distinguished. Initially input operands B0 to B4 are cached in registers and multiplied one after the other with input operands A0 to A4. In the process of multiplication increasingly more registers were required for holding intermediate multiplication results. Completed result words that had been fully accumulated were spilled on the stack in order to free registers for more temporary results. Still starting with the multiplication with input A5, the input operand registers B3 and B4 were required as scratch registers for storing temporaries (multiplication steps 25 to 32 ). Ultimately also B0 had to be discarded. Then, in order to complete words 5 to 6 of the multiplication results (for freeing completed result words by writes to the stack), multiplication of input operands B5 to B7 with A0 to A4 is performed (33 to 47). Subsequently the multiplication result word 7 could be completed after the multiplication of A7 and B0 (Step 48). Finally the multiplications of input words A6 to A7 with B3 to B7 is calculated. Here the values A5 to A7 were cached in registers.

During the multiplication only 8 register spills were necessary for storing the lower-most result words temporarily on the stack. After multiplication the upper 8 result words of 
Table 2: Sequence of executing the 64 partial products of words $A_{i} \times B_{j}$ used for schoolbook multiplication of 256 bit operands.

\begin{tabular}{c|cccccccc} 
& A0 & A1 & A2 & A3 & A4 & A5 & A6 & A7 \\
\hline B0 & 1 & 5 & 10 & 15 & 20 & 25 & 28 & 48 \\
B1 & 0 & 6 & 11 & 16 & 21 & 26 & 29 & 31 \\
B2 & 2 & 7 & 12 & 17 & 22 & 27 & 30 & 32 \\
B3 & 3 & 8 & 13 & 18 & 23 & 49 & 50 & 51 \\
B4 & 4 & 9 & 14 & 19 & 24 & 52 & 53 & 54 \\
B5 & 33 & 36 & 39 & 42 & 45 & 55 & 56 & 57 \\
B6 & 34 & 37 & 40 & 43 & 46 & 58 & 59 & 60 \\
B7 & 35 & 38 & 41 & 44 & 47 & 61 & 62 & 63
\end{tabular}

Table 3: Sequence of executing the partial product words $A_{i} \times A_{j}$ used for schoolbook squaring of 256 bit operands .

\begin{tabular}{c|cccccccc} 
& A0 & A1 & A2 & A3 & A4 & A5 & A6 & A7 \\
\hline A0 & 1 & 2 & & & & & & \\
A1 & 0 & 3 & & & & & & \\
A2 & 5 & 6 & 15 & & & & & \\
A3 & 4 & 11 & 12 & 19 & & & & \\
A4 & 8 & 9 & 16 & 23 & 32 & & & \\
A5 & 7 & 13 & 20 & 24 & 27 & 34 & & \\
A6 & 10 & 17 & 21 & 25 & 28 & 30 & 35 & \\
A7 & 14 & 18 & 22 & 26 & 29 & 31 & 33 & 36
\end{tabular}

the 512 bit multiplication result were reduced within the register set before storing the reduced result back to memory.

\subsubsection{Field squaring for ARM Cortex M4}

For squarings we again make use of a special property of the Cortex-M4 instruction set which allows for two different types of carry chain. Either the ADDS and ADCS instructions may be employed (storing the carry bit in the status register) or UMLAL and UMAAL instructions (which do not modify the carry bit). The latter instructions store carries in full registers. We make use of this by using addition instructions for doubling the off-diagonal parts (with the exception of the product term $\mathrm{A} 1 * \mathrm{~A} 0$ ), while we make use of integrated multiplyaccumulate operations everywhere else.

In comparison to multiplication, we were able to hold more input operands in registers. The following table depicts the sequence $(0 . .36)$ in which the partial products were calculated.

Throughout the calculation we distinguish between off-diagonal multiplication results (which require subsequent doubling) and diagonal multiplication results which were accumulated by use of multiplication instructions. Just as for multiplication, squaring is merged with reduction. This way only 5 register spills to the stack were required for storing intermediate multiplication results. 


\subsection{Implementation of the hash functions}

For the calculation of SHA512 we use the optimized assembly code for the Cortex M0 architecture on both targets. This code fully unrolls the inner loop of the add-rotate-xor algorithm. We also make use of the special instructions for endianness-change. For the Cortex M4, some further speedup would be possible, when exploiting the availability of the "upper" registers. We did, however, stick with the more compact Cortex M0 code also because hashing was not the performance bottle neck.

\section{Experimental results}

In the following sections we will report on experimental results obtained from several different microcontroller targets, nRF51822, nRF52832, STM32F407, STM32F411 and STM32L476. We decided to include figures for all of these instead of selecting one particular chipset since in the course of our analysis, we observed that for the Cortex M4 architecture a major difficulty arises regarding speed benchmarking. Unlike for smaller architectures we observed that the highly target-specific performance of the flash memory subsystem plays a major role for the actual speed.

We did observe the most remarkable effect for the microcontroller STM32L476 targeting specifically ultra-low-power applications. Note that for ultra-low-power operation a suitable compromise between increased microcontroller speed and increased power consumption due to speculative flash accesses has to be found. We attribute our finding that the cycle counts for the analyzed primitives (depending on the power-consumption configuration) could increase by almost $40 \%$ when increasing the clock frequency from $16 \mathrm{MHz}$ to 80 $\mathrm{MHz}$ mainly to such type of optimization. Obviously this makes fair speed benchmarking very difficult.

For the high-performance-family devices STM32F411 and STM32F407 from the same manufacturer, we observed that the influence of the clock frequency on performance is still present, but much smaller. Specifically for the STM32F411 almost no speed reduction was observed also at its highest clock frequency. In addition to flash timing issues, it is worth noting that for the STM32F407 device we observed some further dependence on the RAM memory configuration. This device disposes of so-called core-coupled memory (CCM). The timings reported here were obtained when placing the execution stack to CCM. Speed figures were observed to be significantly faster than when placing the stack in conventional RAM region.

As a result, we conclude that for speed benchmarking for cryptography implementations it is best to compare results obtained at lower clock frequencies. According to our results then some variations between different microcontroller suppliers still do exist, however the resulting values are at least of the same order of magnitude. We suggest, that the STM32F411 as a typical medium-size implementation for IIoT applications might be well suited as kind of reference platform for speed benchmarking.

\subsection{Field arithmetic}

In table 4 the speed results for the field arithmetic are summarized. Despite the mentioned difficulties regarding benchmarking, we come to the conclusion that our field arithmetic is significantly more efficient than the previously best published results on the Cortex M4 microcontroller in [FA17], specifically regarding multiplication and squaring of field elements.

The speedup obtained for the field arithmetic in comparison to reports from Aranha and Fujii in [FA17] in our opinion might stem from the following differences. Firstly for multiplication and squaring we did merge multiplication and squaring functions with reductions. This allowed us to hold more operands in registers. Secondly with the realized 
Table 4: Field arithmetic on different targets at different frequencies $\mathrm{f}(/ \mathrm{MHz})$. Columns $* A_{0}\left(+* A_{0}\right)$ contain clock cycles for multiplication with the field constant $A_{0}=121666$ and merged addition and multiplication $x+y * A_{0}$. Cycle count for the nRF51 target was obtained with the CLANG compiler with compile switch -O2 while for the ST Microelectronics microcontroller we did use GCC 4.9.2 with optimization setting -O2.

\begin{tabular}{l|c|c|c|c|c|c|c|l} 
Target & $\mathrm{f}$ & $x+y$ & $x-y$ & $* A_{0}$ & $+* A_{0}$ & $x^{2}$ & $x * y$ & \\
\hline nRF51822 & 16 & 120 & 147 & 193 & - & 998 & 1478 & $\mathbb{F}_{\left(2^{255}-19\right)}$, this work \\
\hline STM32F411 & $?$ & 73 & 77 & 129 & - & 563 & 631 & $\mathbb{F}_{\left(2^{255}-19\right)},[\mathrm{DSS} 16]$ \\
MK20DX & 72 & 86 & 86 & 76 & - & 252 & 276 & $\mathbb{F}_{\left(2^{255}-19\right)},[$ FA17] \\
STM32F411 & 16 & 55 & 72 & - & 58 & 153 & 222 & $\mathbb{F}_{\left(2^{255}-19\right)}$, this work \\
STM32L476 & 16 & 52 & 65 & - & 55 & 153 & 220 & $\mathbb{F}_{\left(2^{255}-19\right), \text { this work }}$ \\
STM32L476 & 80 & 95 & 124 & - & 95 & 168 & 237 & $\mathbb{F}_{\left(2^{255}-19\right), \text { this work }}$ \\
nRF52832 & 64 & 62 & 70 & - & 65 & 162 & 229 & $\mathbb{F}_{\left(2^{255}-19\right), \text { this work }}$ \\
STM32F407 & 84 & 56 & 74 & & 56 & 155 & 223 & $\mathbb{F}_{\left(2^{255}-19\right)}$, this work \\
\hline STM32F407 & 84 & 86 & - & - & - & 215 & 358 & $\mathbb{F}_{\left(2^{127}-1\right)^{2}}\left[\mathrm{LLP}^{+} 17\right]$ \\
\hline
\end{tabular}

level of optimization regarding multiplication and squaring, the performance of addition and subtraction within the X25519 calculations starts becoming important as well. For these simpler operations call overhead becomes significant and use of inline assembly functions highly beneficial.

For the purpose of comparison, we also did add timings for the field $\mathbb{F}_{\left(2^{127}-1\right)^{2}}$ as used in the construction Four $\mathbb{Q}$ in $\left[\mathrm{CL} 15, \mathrm{LLP}^{+} 17\right]$. Note that our timings for $\mathbb{F}_{\left(2^{255}-19\right)}$ are significantly faster despite the fact that the group order is comparable. As a result, we expect that a large fraction of the algorithmic speedup that is made possible by the endomorphisms of Four $\mathbb{Q}$ is lost by less efficient field arithmetic in [LLP+17]. Note, that the most relevant figure for the speed of Diffie-Hellman on Four $\mathbb{Q}$ is field multiplication, where the difference to our results is particularly large. We have reviewed the field operations in $\left[\mathrm{LLP}^{+} 17\right]$ and believe that significant further speedups should be possible also for Four $\mathbb{Q}$. For field multiplication, for instance, we believe that a speedup in the range of 25 percent might be feasible in comparison to $\left[\mathrm{LLP}^{+} 17\right]$ when using the methods described in this paper.

\subsection{X25519 Diffie-Hellman}

In table 5 we summarize the results for the X25519 function for different microcontrollers and different clock frequencies. Our fastest result for X25519 on the M4 executes in as little as 609.779 cycles and is, thus, roughly 3 and 2.5 times faster than the reports in [dG15] (1816351) and [DSS16] (1563852) respectively and also significantly faster than the previously fastest result (907.240 cycles) from [FA17]. It is worth noting that in contrast to [FA17] we did use (in line with [HL17]) constant-time swaps of pointers instead of swapping full field elements. Note that for internal memories of Cortex M4 and M0 access timing is deterministic. When swapping pointers we expect both, more speed and less side-channel leakage. Note however, that our implementation requires (unlike [FA17]) to be run with using internal RAM memory with constant access times. Our implementation optionally also allows for swapping field elements instead of pointers. According to our own measurements the penalty of doing so accounts roughly for additional 50.000 clock cycles.

Again we also have added speed benchmarks for Four $\mathbb{Q}$ from $\left[\mathrm{LLP}^{+} 17\right]$ for reference. Note that comparing of the fundamentally different algorithms X25519 and Diffie-Hellman 
Table 5: Speed of X25519 scalar multiplication (Four $\mathbb{Q}$ ) (on different targets, clock and memory configurations). The timings marked with $(p)$ were obtained with enabled flash pre-fetch engines which increase current consumption.

\begin{tabular}{l|c|c|l} 
Target & $\mathrm{f} / \mathrm{MHz}$ & $\mathrm{X} 25519$ & \\
\hline nRF51822 & 16 & $3,474,201$ & this work \\
\hline STM32F411 & $?$ & $1,816,351$ & {$[\mathrm{dG15}]$} \\
STM32F411 & $?$ & $1,563,852$ & {$[\mathrm{DSS} 16]$} \\
MK20DX & 72 & 907,240 & {$[$ FA17] } \\
STM32L476 & $16 ; 80^{(p)} ; 80$ & 609,$779 ; 857,002 ; 971,272$ & this work \\
nRF52832 & 64 & 634,567 & this work \\
STM32F411 & $16 ; 100^{(p)} ; 100$ & 625,$347 ; 625,449 ; 734,554$ & this work \\
STM32F407 & $16 ; 84(\mathrm{p}) ; 168^{(p)} ; 168$ & 625,$358 ; 626,719 ; 655,891 ; 847,048$ & this work \\
\hline STM32F407 & $84^{(p)}$ & $542,900($ FourQ $)$ & {$\left[\mathrm{LLP}^{+} 17\right]$}
\end{tabular}

Table 6: Cycle counts for the nRF51822 Cortex M0 (STM32F411 Cortex M4) microcontrollers running at $16 \mathrm{MHz}$ for SHA512, Elligator2, a X25519 Montgomery ladder step (LS), Inversion $(1 / x)$ and a complete partially augmented AuCPace protocol run.

\begin{tabular}{r|c|c|c|c|c} 
Target & SHA512 & Elligator2 & LS & $1 / x$ & AuCPace \\
\hline nRF51822 & 21,564 & 289,276 & 13,521 & 258,291 & $7,345,820$ \\
\hline STM32F411 & 21,130 & 46,032 & 3,163 & 42,590 & $1,351,381$
\end{tabular}

on Four $\mathbb{Q}$ is difficult. For instance, when using the endomorphisms in Four $\mathbb{Q}$ quite large tables in RAM are required (required stack size is unfortunately not reported in $\left.\left[\mathrm{LLP}^{+} 17\right]\right)$. Also note that the code size is about a factor of three larger than for our X25519 implementation. Despite the fact that our X25519 implementation (ca. 625,500 cycles) is much more adapted to small targets, our observed speed is very competitive in comparison to the reported result for Diffie-Hellman on Four $\mathbb{Q}(542,900$ cycles including point decompression).

\subsection{Partially augmented AuCPace25519}

We have implemented AuCPace by using an asynchronous execution engine as suggested in [HL17]. Table 6 summarizes the speed results for individual substeps for the Cortex M0 (nRF51822) and different Cortex M4 microcontrollers.

We observed a speedup of roughly a factor of two in comparison to the results of [HL17] regarding the Elligator2 substep. In [HL17] the Elligator2 mapping algorithm was calculated by use of two separate exponentiations for inversion and calculation of the Legendre symbol $\chi$. In our work, we make use of the inverse square root algorithm for calculating Elligator2 with one single field exponentiation. In total this accounts for roughly 4 percent of a speedup regarding the balanced PACE (CPace) protocol runs on the Cortex M0.

Our results for the Cortex-M4 microcontroller family are faster by a factor of 5.4 in comparison to the Cortex M0, showing that this microcontroller architecture with its signalprocessing instructions is by far better suited and likely also much more power-efficient for implementing complex asymmetric cryptography.

In table 7 the memory consumptions for the asynchronous execution object ACE from [HL17] and the stand-alone algorithm X25519 are summarized. The figures for the ACE object also include a Salsa20-20 based pseudo-random-number generator and the 
Table 7: Memory consumption in bytes for asynchronized implementation of AuCPace (ACE) and X25519 for Cortex M0 and M4 microcontrollers. Results were obtained with arm-none-eabi-gcc-O2 (gcc version 4.9.3). RAM consumption is separated in static memory (stack memory) respectively.

\begin{tabular}{l|c|c||c|c|l} 
Target & RAM & ROM & RAM & ROM & \\
Target & ACE & ACE & X25519 & X25519 & \\
\hline Cortex-M0 & $264(396)$ & 11252 & $0(572)$ & 6108 & this work \\
\hline Cortex-M4 & $264(268)$ & 8896 & $0(444)$ & 3324 & this work \\
Cortex-M4 & & & & 4152 & [FA17] \\
Cortex-M4 & & & & 3786 & [DSS16] \\
\hline
\end{tabular}

implementation for SHA512. For the Cortex M4 version, the total RAM requirement amounts to 532 bytes including static memory and stack. The stand-alone synchronous X25519 implementation (no state in static memory) for the Cortex M4 needs 444 bytes of stack memory and 3,324 bytes of flash and improves, thus, upon previous work [DSS16, FA17].

All of our code avoids secret-dependent branches and is, thus, executing in constant time on target-platforms with deterministic RAM memory access timings, such as typically found in ARM Cortex M0 and M4 microcontrollers.

\section{Discussion and conclusion}

In this paper we have presented a comprehensive analysis regarding possible optimizations for verifier-based password-authenticated key exchange for the setting of resourceconstrained servers. Our analysis did cover all of protocol design, protocol security proof, algorithmic optimization regarding group operations and field arithmetic and assembly-level fine-tunings.

Our construction allows for particular advantages in IIoT settings where a large number of small server nodes should be expected to operate with the same passwords, such as, e.g., the case in industrial plants. In addition to the conventional notion of verifier-based PAKE, our construction also allows for a partial augmentation operation mode that essentially halves the server's computational complexity of the password verification step.

Our construction with full augmentation imposes a complexity of four exponentiations in total on the server, one of which could be pre-computed prior to each login. The user-perceived login delay, thus is governed by the time consumed for calculating three scalar multiplications.

Our construction is two exponentiations faster for the server than all previously known verifier-based PAKE constructions when instantiated in its partially augmented variant. In the setting of [HL17] where one scalar multiplication accounts for about two seconds this results in a clearly perceivable usability gain in comparison to previously known protocols requiring at least three scalar multiplications.

Moreover our construction inherently allows for using strong memory-hard password hashing also on small servers since the costly memory-consuming operations are deferred to the clients.

The composability of the AuCPace security guarantees facilitates security analysis for use of AuCPace, e.g., as a building block in larger constructions, such as a centralized ticket-based user-credential distribution framework for industrial plants.

In contrast to most previous Diffie-Hellman based V-PAKE constructions with analysis in the UC framework, our security proof provides guarantees also in the stronger 
fully adaptive adversary model which allows for corruptions at any time during session establishment.

Finally, we have presented performance benchmarks of an instantiation targeting common microcontroller platforms coined AuCPace25519 which instantiates our protocol with using the primitives X25519, Elligator2 and SHA512.

The protocol runs in only $1,351,381(7,345,820)$ cycles for a partially augmented protocol run on an ARM Cortex-M4 (M0) microcontroller respectively. On the M4 AuCPace requires only 8896 (532) bytes of flash (RAM) memory. There the X25519 Diffie-Hellman protocol sub-step executes in as little as 609,779 cycles. Our implementation, thus, sets up new speed records for both, (V)-PAKE protocols and X25519 Diffie-Hellman key exchange on this important embedded CPU architecture platform. This illustrates also that on the Cortex M4 X25519 could be implemented very competitively, even in comparison to the best currently published implementations that exploit additional structure in elliptic curves, such as endomorphisms.

Summing up, we believe that all of the individual components presented in this paper in combination might yield a solution particularly tailored for the needs of real-world resource-constrained IIoT environments, such as notably intrinsically safe power limited industrial instrumentation.

\section{Acknowledgements}

The authors would like to thank the anonymous referees for their helpful comments and advice and their great care with the manuscript. We also acknowledge inspiring discussions with Daniel Rausch, Ralf Küsters, Denis Kügler, Marc Fischlin, Mike Hamburg and Peter Schwabe.

\section{References}

[ACCP08] Michel Abdalla, Dario Catalano, Céline Chevalier, and David Pointcheval. Efficient two-party password-based key exchange protocols in the UC framework. In Topics in Cryptology - CT-RSA 2008, The Cryptographers' Track at the RSA Conference 2008, San Francisco, CA, USA, April 8-11, 2008. Proceedings, pages 335-351. 2008.

[AFP05] Michel Abdalla, Pierre-Alain Fouque, and David Pointcheval. Password-based authenticated key exchange in the three-party setting. In Serge Vaudenay, editor, Public Key Cryptography - PKC 2005, 8th International Workshop on Theory and Practice in Public Key Cryptography, Les Diablerets, Switzerland, January 23-26, 2005, Proceedings, volume 3386 of Lecture Notes in Computer Science, pages 65-84. Springer, 2005.

[AP05] Michel Abdalla and David Pointcheval. Simple password-based encrypted key exchange protocols. In Alfred Menezes, editor, Topics in Cryptology - CT-RSA 2005, The Cryptographers' Track at the RSA Conference 2005, San Francisco, CA, USA, February 14-18, 2005, Proceedings, volume 3376 of Lecture Notes in Computer Science, pages 191-208. Springer, 2005.

[BDKJ16] Alex Biryukov, Daniel Dinu, Dmitry Khovratovich, and Simon Josefsson. The memory-hard argon2 password hash and proof-of-work function. Technical report, Internet-Draft draft-irtf-cfrg-argon2-00, Internet Engineering Task Force, 2016. Work in Progress, 2016. 
$\left[\mathrm{BDL}^{+} 11\right]$ Daniel J. Bernstein, Niels Duif, Tanja Lange, Peter Schwabe, and Bo-Yin Yang. High-speed high-security signatures. In Bart Preneel and Tsuyoshi Takagi, editors, Cryptographic Hardware and Embedded Systems - CHES 2011 - 13th International Workshop, Nara, Japan, September 28 - October 1, 2011. Proceedings, volume 6917 of Lecture Notes in Computer Science, pages 124-142. Springer, 2011.

[Ber06] Daniel J. Bernstein. Curve25519: new Diffie-Hellman speed records. In Moti Yung, Yevgeniy Dodis, Aggelos Kiayias, and Tal Malkin, editors, Public Key Cryptography - PKC 2006, volume 3958 of Lecture Notes in Computer Science, pages 207-228. Springer-Verlag Berlin Heidelberg, 2006. http://cr.yp.to/ papers.html\#curve25519.

[Ber14] Daniel J. Bernstein. 25519 naming. Posting to the CFRG mailing list, 2014. https://www.ietf .org/mail-archive/web/cfrg/current/msg04996. html.

[BFK09] Jens Bender, Marc Fischlin, and Dennis Kügler. Security analysis of the PACE key-agreement protocol. In Pierangela Samarati, Moti Yung, Fabio Martinelli, and Claudio Agostino Ardagna, editors, Information Security, 12th International Conference, ISC 2009, Pisa, Italy, September 7-9, 2009. Proceedings, volume 5735 of Lecture Notes in Computer Science, pages 33-48. Springer, 2009.

[BHKL13] Daniel J. Bernstein, Mike Hamburg, Anna Krasnova, and Tanja Lange. Elligator: elliptic-curve points indistinguishable from uniform random strings. In Ahmad-Reza Sadeghi, Virgil D. Gligor, and Moti Yung, editors, 2013 ACM SIGSAC Conference on Computer and Communications Security, CCS'13, Berlin, Germany, November 4-8, 2013, pages 967-980. ACM, 2013.

[BL19] Daniel J. Bernstein and Tanja Lange. SafeCurves: Choosing safe curves for elliptic-curve cryptography. Definition of Twist security. (accessed on 15 January 2019), 2019. https://safecurves.cr.yp.to/twist.html.

[BLR04] Boaz Barak, Yehuda Lindell, and Tal Rabin. Protocol initialization for the framework of universal composability. Cryptology ePrint Archive, Report 2004/006, 2004. https://eprint.iacr.org/2004/006.

[BM92] Steven M. Bellovin and Michael Merritt. Encrypted key exchange: passwordbased protocols secure against dictionary attacks. In 1992 IEEE Computer Society Symposium on Research in Security and Privacy, Oakland, CA, USA, May 4-6, 1992, pages 72-84. IEEE Computer Society, 1992.

[BMP00] Victor Boyko, Philip D. MacKenzie, and Sarvar Patel. Provably secure passwordauthenticated key exchange using diffie-hellman. In Preneel [Pre00], pages $156-171$

[BPR00] Mihir Bellare, David Pointcheval, and Phillip Rogaway. Authenticated key exchange secure against dictionary attacks. In Preneel [Pre00], pages 139-155.

[BRSS18] José Becerra, Peter Y. A. Ryan, Petra Sala, and Marjan Skrobot. An offline dictionary attack against zkpake protocol. In Proceedings of the 11th ACM Conference on Security 8 Privacy in Wireless and Mobile Networks, WiSec 2018, Stockholm, Sweden, June 18-20, 2018, pages 291-292, 2018.

[Can00] Ran Canetti. Universally composable security: A new paradigm for cryptographic protocols. IACR Cryptology ePrint Archive, 2000:67, 2000. 
[Can01] Ran Canetti. Universally composable security: A new paradigm for cryptographic protocols. In 42nd Annual Symposium on Foundations of Computer Science, FOCS 2001, 14-17 October 2001, Las Vegas, Nevada, USA, pages 136-145. IEEE Computer Society, 2001.

[CGIP12] Jean-Sébastien Coron, Aline Gouget, Thomas Icart, and Pascal Paillier. Supplemental access control (PACE v2): Security analysis of PACE integrated mapping. In David Naccache, editor, Cryptography and Security: From Theory to Applications - Essays Dedicated to Jean-Jacques Quisquater on the Occasion of His 65th Birthday, volume 6805 of Lecture Notes in Computer Science, pages 207-232. Springer, 2012.

$\left[\mathrm{CHK}^{+} 05\right]$ Ran Canetti, Shai Halevi, Jonathan Katz, Yehuda Lindell, and Philip D. MacKenzie. Universally composable password-based key exchange. In Advances in Cryptology - EUROCRYPT 2005, 24th Annual International Conference on the Theory and Applications of Cryptographic Techniques, Aarhus, Denmark, May 22-26, 2005, Proceedings, pages 404-421, 2005.

[CKS08] David Cash, Eike Kiltz, and Victor Shoup. The twin diffie-hellman problem and applications. In Nigel P. Smart, editor, Advances in Cryptology - EUROCRYPT 2008, 27th Annual International Conference on the Theory and Applications of Cryptographic Techniques, Istanbul, Turkey, April 13-17, 2008. Proceedings, volume 4965 of Lecture Notes in Computer Science, pages 127-145. Springer, 2008 .

[CL15] Craig Costello and Patrick Longa. Fourı: Four-dimensional decompositions on a ॥-curve over the mersenne prime. In Tetsu Iwata and Jung Hee Cheon, editors, Advances in Cryptology - ASIACRYPT 2015 - 21st International Conference on the Theory and Application of Cryptology and Information Security, Auckland, New Zealand, November 29 - December 3, 2015, Proceedings, Part I, volume 9452 of Lecture Notes in Computer Science, pages 214-235. Springer, 2015.

[CR03] Ran Canetti and Tal Rabin. Universal composition with joint state. In Dan Boneh, editor, Advances in Cryptology - CRYPTO 2003, 23rd Annual International Cryptology Conference, Santa Barbara, California, USA, August 17-21, 2003, Proceedings, volume 2729 of Lecture Notes in Computer Science, pages 265-281. Springer, 2003.

[dG15] Wouter de Groot. A Performance Study of X25519 on Cortex-M3 and M4. $\mathrm{PhD}$ thesis, Master thesis, Eindhoven University of Technology (Sep 2015), 2015.

[DHH $\left.{ }^{+} 15\right]$ Michael Düll, Björn Haase, Gesine Hinterwälder, Michael Hutter, Christof Paar, Ana Helena Sánchez, and Peter Schwabe. High-speed curve25519 on 8-bit, 16-bit, and 32-bit microcontrollers. Des. Codes Cryptography, 77(2-3):493-514, 2015 .

[DSS16] Fabrizio De Santis and Georg Sigl. Towards side-channel protected X25519 on ARM Cortex-M4 processors. In Proceedings of Software performance enhancement for encryption and decryption, and benchmarking, Utrecht, The Netherlands., 2016.

[EKSS09] John Engler, Chris Karlof, Elaine Shi, and Dawn Song. Is it too late for PAKE? In Proceedings of the IEEE Web 2.0 Security and Privacy Workshop, volume 5, page 17, 2009. 
[FA17] Hayato Fujii and Diego F Aranha. Curve25519 for the Cortex-M4 and beyond. Progress in Cryptology-LATINCRYPT, 2017.

[GMR06] Craig Gentry, Philip D. MacKenzie, and Zulfikar Ramzan. A method for making password-based key exchange resilient to server compromise. In Cynthia Dwork, editor, Advances in Cryptology - CRYPTO 2006, 26th Annual International Cryptology Conference, Santa Barbara, California, USA, August 20-24, 2006, Proceedings, volume 4117 of Lecture Notes in Computer Science, pages 142-159. Springer, 2006.

[Gre18] Matthew Green. Let's talk about PAKE, 2018. https://blog. cryptographyengineering.com/2018/10/19/lets-talk-about-pake/.

[Ham12] Mike Hamburg. Fast and compact elliptic-curve cryptography. IACR Cryptology ePrint Archive, 2012:309, 2012.

[Ham17] Mike Hamburg. ISR trick for elligator2. Posting to the Curves mailing list, 2017. https://moderncrypto.org/mail-archive/curves/2017/000939.html.

[HL17] Björn Haase and Benoît Labrique. Making password authenticated key exchange suitable for resource-constrained industrial control devices. In Wieland Fischer and Naofumi Homma, editors, Cryptographic Hardware and Embedded Systems - CHES 2017 - 19th International Conference, Taipei, Taiwan, September 25-28, 2017, Proceedings, volume 10529 of Lecture Notes in Computer Science, pages 346-364. Springer, 2017.

[HL18] Björn Haase and Benoît Labrique. Repository for optimized X25519 field arithmetics code for ARM cortex M4 microcontrollers, 2018. https://github . com/BjoernMHaase/fe25519.

[HR10] Feng Hao and Peter Ryan. J-PAKE: authenticated key exchange without PKI. Trans. Computational Science, 11:192-206, 2010.

[HS14] Feng Hao and Siamak Fayyaz Shahandashti. The SPEKE protocol revisited. In Liqun Chen and Chris J. Mitchell, editors, Security Standardisation Research First International Conference, SSR 2014, London, UK, December 16-17, 2014. Proceedings, volume 8893 of Lecture Notes in Computer Science, pages 26-38. Springer, 2014.

[Jab96] David P. Jablon. Strong password-only authenticated key exchange. Computer Communication Review, 26(5):5-26, 1996.

[Jab97] David P. Jablon. Extended password key exchange protocols immune to dictionary attacks. In 6th Workshop on Enabling Technologies (WET-ICE '97), Infrastructure for Collaborative Enterprises, 18-20 June 1997, MIT, Cambridge, MA, USA, Proceedings, pages 248-255, 1997.

[JKX18] Stanislaw Jarecki, Hugo Krawczyk, and Jiayu Xu. OPAQUE: an asymmetric PAKE protocol secure against pre-computation attacks. In Jesper Buus Nielsen and Vincent Rijmen, editors, Advances in Cryptology - EUROCRYPT 2018 - 37th Annual International Conference on the Theory and Applications of Cryptographic Techniques, Tel Aviv, Israel, April 29 - May 3, 2018 Proceedings, Part III, volume 10822 of Lecture Notes in Computer Science, pages 456-486. Springer, 2018. 
[KR17] Ralf Küsters and Daniel Rausch. A framework for universally composable diffiehellman key exchange. In 2017 IEEE Symposium on Security and Privacy, SP 2017, San Jose, CA, USA, May 22-26, 2017, pages 881-900. IEEE Computer Society, 2017.

[Kra05] Hugo Krawczyk. HMQV: A high-performance secure diffie-hellman protocol. In Victor Shoup, editor, Advances in Cryptology - CRYPTO 2005: 25th Annual International Cryptology Conference, Santa Barbara, California, USA, August 14-18, 2005, Proceedings, volume 3621 of Lecture Notes in Computer Science, pages 546-566. Springer, 2005.

[Kra18] Hugo Krawczyk. The opaque asymmetric pake protocol, 2018. https://tools . ietf.org/pdf/draft-krawczyk-cfrg-opaque-00.pdf.

[KTR13] Ralf Kuesters, Max Tuengerthal, and Daniel Rausch. The IITM model: a simple and expressive model for universal composability. Cryptology ePrint Archive, Report 2013/025, 2013. https://eprint.iacr.org/2013/025.

[LL97] Chae Hoon Lim and Pil Joong Lee. A key recovery attack on discrete log-based schemes using a prime order subgroupp. In Burton S. Kaliski Jr., editor, Advances in Cryptology - CRYPTO '97, 17th Annual International Cryptology Conference, Santa Barbara, California, USA, August 17-21, 1997, Proceedings, volume 1294 of Lecture Notes in Computer Science, pages 249-263. Springer, 1997.

[LLM07] Brian A. LaMacchia, Kristin E. Lauter, and Anton Mityagin. Stronger security of authenticated key exchange. In Willy Susilo, Joseph K. Liu, and Yi Mu, editors, Provable Security, First International Conference, ProvSec 2007, Wollongong, Australia, November 1-2, 2007, Proceedings, volume 4784 of Lecture Notes in Computer Science, pages 1-16. Springer, 2007.

[LLP $\left.{ }^{+} 17\right]$ Zhe Liu, Patrick Longa, Geovandro C. C. F. Pereira, Oscar Reparaz, and Hwajeong Seo. Fourq on embedded devices with strong countermeasures against side-channel attacks. IACR Cryptology ePrint Archive, 2017:434, 2017.

[LW15] Hanwook Lee and Dongho Won. Prevention of exponential equivalence in simple password exponential key exchange (SPEKE). Symmetry, 7(3):1587-1594, 2015.

[Mac01] Philip MacKenzie. On the security of the SPEKE password-authenticated key exchange protocol. IACR Cryptology ePrint Archive, 2001:57, 2001.

[MRA15] Karina Mochetti, Amanda C Davi Resende, and Diego F Aranha. zkpake: A simple augmented PAKE protocol. In Proceedings of the Brazilian Symposium on Information and Computational Systems Security (SBSeg), 2015.

[PJ12] Colin Percival and Simon Josefsson. The scrypt password-based key derivation function., 2012. http://tools.ietf.org/html/josefsson-scrypt-kdf-00. txt.

[Pre00] Bart Preneel, editor. Advances in Cryptology - EUROCRYPT 2000, International Conference on the Theory and Application of Cryptographic Techniques, Bruges, Belgium, May 14-18, 2000, Proceeding, volume 1807 of Lecture Notes in Computer Science. Springer, 2000.

[PW17] David Pointcheval and Guilin Wang. VTBPEKE: verifier-based two-basis password exponential key exchange. In Ramesh Karri, Ozgur Sinanoglu, Ahmad-Reza Sadeghi, and Xun Yi, editors, Proceedings of the 2017 ACM 
on Asia Conference on Computer and Communications Security, AsiaCCS 2017, Abu Dhabi, United Arab Emirates, April 2-6, 2017, pages 301-312. ACM, 2017.

[RS17] Joost Renes and Benjamin Smith. qdsa: Small and secure digital signatures with curve-based diffie-hellman key pairs. In Tsuyoshi Takagi and Thomas Peyrin, editors, Advances in Cryptology - ASIACRYPT 2017 - 23rd International Conference on the Theory and Applications of Cryptology and Information Security, Hong Kong, China, December 3-7, 2017, Proceedings, Part II, volume 10625 of Lecture Notes in Computer Science, pages 273-302. Springer, 2017.

[Sho97] Victor Shoup. Lower bounds for discrete logarithms and related problems. In Walter Fumy, editor, Advances in Cryptology - EUROCRYPT '97, International Conference on the Theory and Application of Cryptographic Techniques, Konstanz, Germany, May 11-15, 1997, Proceeding, volume 1233 of Lecture Notes in Computer Science, pages 256-266. Springer, 1997.

[SKI10] SeongHan Shin, Kazukuni Kobara, and Hideki Imai. Security proof of AugPAKE. IACR Cryptology ePrint Archive, 2010:334, 2010.

[SOAA15] Stanislav Smyshlyaev, Igor B. Oshkin, Evgeniy K. Alekseev, and Liliya R. Ahmetzyanova. On the security of one password authenticated key exchange protocol. IACR Cryptology ePrint Archive, 2015:1237, 2015.

[Wu98] Thomas D. Wu. The secure remote password protocol. In Proceedings of the Network and Distributed System Security Symposium, NDSS 1998, San Diego, California, USA, pages 97-111. The Internet Society, 1998.

[Zha04] Muxiang Zhang. Analysis of the SPEKE password-authenticated key exchange protocol. IEEE Communications Letters, 8(1):63-65, 2004. 


\section{A Notes regarding short Weierstrass curves}

Our construction shares with [JKX18] the requirement that an efficient hashing to group elements must be available for the elliptic curve's point group. Unfortunately, this is not always the case for important established curves, namely regarding standards using the short Weierstrass form. In order to circumvent this problem, as an alternative in [PW17] a construction TBPEKE based on two base points and an additional scalar multiplication has been suggested by Pointcheval and Wang. Note that this construction is very similar to the balanced sub-protocol CPace presented in this paper.

In this appendix we deal with the question, whether the TBPEKE construction could also be used instead of CPace as a balanced sub-protocol component for AuCPace. I.e. the question is whether the TBPEKE construction could also be proven secure in the UC model. In our opinion, this answer could be given affirmatively. However, unfortunately, our UC security proof technique that allowed for fully adaptive adversaries could probably not be carried out for TBPEKE because of a technical commitment problem within the Diffie-Hellman step. However, we come to the conclusion, that the balanced sub-step of TBPEKE could be proven secure also in the UC framework, when considering a weaker static adversary model as used for most other security proofs of efficient constructions in the UC framework such as [GMR06].

I.e. for implementations forced to use older short Weierstrass curves, we suggest to replace our technique for the calculation of the ephemeral generator $G$ as $G=$ Map2Point $\left(\mathrm{H}_{1}(P R S)\right)$ by the TBPEKE equivalent of $G=A+C^{\mathrm{H}_{1}(P R S)}$. The essential property (as pointed out also in [BFK09]) is that the discrete logarithm of $G$ must be unknown for both, honest parties and the adversaries.

Note that for any TBPEKE-based construction we see as important pre-requisite that the "nothing-upon-my-sleeve" problem related to the secrecy of the discrete log of the points $C$ and $A$ needs to be resolved in a trustworthy way.

Here we make the following suggestion. For any of the older short Weierstrass form elliptic curves we suggest to determine the curve points $A$ and $C$ by the following algorithm. For the point $A(C)$ we suggest to first take the packed little-endian encoding of the standardized curve's base point $x(y)$ coordinate and calculate $\tilde{x}_{A}=\operatorname{SHA} 512(x)\left(\tilde{x}_{C}=\right.$ SHA512(y)). When doing so, there is a non-negligible probability that the $x$-coordinates $\tilde{x}_{A}$ and $\tilde{x}_{C}$ actually correspond to the $x$-coordinate of a point on the twist or possibly on a small subgroup. In this case we suggest to increment the $x$ coordinate step by step by one until a point on the cryptographic group is returned. We then suggest to choose the one out of two $y$-coordinate candidates $y_{A}$ and $y_{C}$ such that the least-significant bit of the $y$-coordinate is zero.

Based on the assumption that no common mathematical structure is shared between the respective short Weierstrass curve and the Add-Rotate-XOR (ARX) algorithm SHA512, we conclude that it is justified to conjecture the secrecy of the discrete logarithms of $A$ and $C$. 


\section{B Notes regarding UC security of the PACE protocol vari- ant from [HL17]}

The protocol in [HL17] is closely related to the protocol CPace presented in this paper. This protocol and the specific optimization steps were not yet analyzed within the UC framework. The main difference to CPace stems from the strategy used for circumventing the patents on SPEKE.

While we do not detail a full UC security proof for this protocol, we nevertheless would like to sketch the necessary steps for executing it. In the notation of [HL17] the password $p w$ corresponds to a password-based key $\pi$ generated, e.g. by hashing the password. The difference of [HL17] to CPace essentially is that the calculation of the password related string $P R S$ involves an additional symmetric encryption, not actually needed for securely implementing the protocol. In order to cover the patent circumvention protocol, we suggest to proceed as follows:

- Firstly we use the encrypted version of the messages $s$ together with the nonce value and the message $t$ that are exchanged at the beginning of the protocol for deriving the session id needed for the UC framework.

- We then use the symmetric Salsa20-20 primitive on the password in order to generate the XOR pad used in [HL17] and modify the definition of the password-related string $P R S$ according to the patent circumvention construction $s \| t$.

- In order to fend off relay attacks, it will be mandatory to incorporate identifiers for the parties (corresponding to the CI of CPace) into the input to the Map2Point function $(\mathrm{H}(s \| t))$ such that not only the password is authenticated but also the client and server identities. This could e.g. be done by incorporating a channel identifier component into input parameter $\pi$ (the password-derived key) used in [HL17].

- We then prove that the password dependent string $(s \| t)$ generated this way matches iff the same password parameter $\pi$ was used by both, server and client, ensuring that the password and the identities match. For this step, we essentially need the property that the entropy of $\pi$ is preserved when extracting a random stream from $\pi$ and nonce value by using Salsa20-20.

- The rest of the proof could then be executed by the same procedures as used in this paper. (Note that the explicit authentication step involving generation of several authenticators by one single run of SHA512 is not mandatory for securely implementing $\mathcal{F}_{\text {pwKE. }}$ ) 